\title{
Single metal-poor ultra compact dwarf galaxy at one kiloparsec distance from the low-mass elliptical galaxy FCC $47^{\star, \star \star}$
}

\author{
Katja Fahrion ${ }^{1}$, Iskren Georgiev ${ }^{2}$, Michael Hilker ${ }^{1}$, Mariya Lyubenova ${ }^{1}$, Glenn van de Ven ${ }^{3}$, Mayte Alfaro-Cuello ${ }^{2}$,
} Enrico M. Corsini ${ }^{4,5}$, Marc Sarzi ${ }^{6,7}$, Richard M. McDermid ${ }^{8}$, and Tim de Zeeuw ${ }^{9,10}$

${ }^{1}$ European Southern Observatory, Karl Schwarzschild Straße 2, 85748 Garching bei München, Germany e-mail: kfahrion@eso.org

2 Max-Planck-Institut für Astronomie, Königstuhl 17, 69117 Heidelberg, Germany

3 Department of Astrophysics, University of Vienna, Türkenschanzstrasse 17, 1180 Wien, Austria

4 Dipartimento di Fisica e Astronomia “G. Galilei”, Universitá di Padova, Vicolo dell'Osservatorio 3, 35122 Padova, Italy

5 INAF-Osservatorio Astronomico di Padova, Vicolo dell'Osservatorio 5, 35122 Padova, Italy

6 Armagh Observatory and Planetarium, College Hill, Armagh BT61 9DG, UK

7 Centre for Astrophysics Research, University of Hertfordshire, College Lane, Hatfield AL10 9AB, UK

8 Department of Physics and Astronomy, Macquarie University, North Ryde, NSW 2109, Australia

9 Sterrewacht Leiden, Leiden University, Postbus 9513, 2300, RA Leiden, The Netherlands

10 Max-Planck-Institut für extraterrestrische Physik, Gießenbachstraße 1, 85748 Garching bei München, Germany

Received 21 December 2018 / Accepted 1 April 2019

\begin{abstract}
Context. Photometric surveys of galaxy clusters have revealed a large number of ultra compact dwarfs (UCDs) around predominantly massive elliptical galaxies. Their origin is still debated as some UCDs are considered to be the remnant nuclei of stripped dwarf galaxies while others seem to mark the high-mass end of the star cluster population.

Aims. We aim to characterize the properties of a UCD found at very close projected distance $\left(r_{\text {proj }}=1.1 \mathrm{kpc}\right)$ from the centre of the low-mass $\left(M \sim 10^{10} M_{\odot}\right)$ early-type galaxy FCC 47 . This is a serendipitous discovery from MUSE adaptive optics science verification data. We explore the potential origin of this UCD as either a massive cluster or the remnant nucleus of a dissolved galaxy.

Methods. We used archival Hubble Space Telescope data to study the photometric and structural properties of FCC 47-UCD1. In the MUSE data, the UCD is unresolved, but we used its spectrum to determine the radial velocity and metallicity.

Results. The surface brightness of FCC 47-UCD1 is best described by a single King profile with low concentration $C=R_{\mathrm{t}} / R_{\mathrm{c}} \sim 10$ and large effective radius $\left(r_{\mathrm{eff}}=24 \mathrm{pc}\right)$. Its integrated magnitude and blue colour $\left(M_{g}=-10.55 \mathrm{mag},(g-z)=1.46 \mathrm{mag}\right)$ combined with a metallicity of $[\mathrm{M} / \mathrm{H}]=-1.12 \pm 0.10 \mathrm{dex}$ and an age $>8 \mathrm{Gyr}$ obtained from the full fitting of the MUSE spectrum suggests a stellar population mass of $M_{*}=4.87 \times 10^{6} M_{\odot}$. The low S/N of the MUSE spectrum prevents detailed stellar population analysis. Due to the limited spectral resolution of MUSE, we can only give an upper limit on the velocity dispersion $\left(\sigma<17 \mathrm{~km} \mathrm{~s}^{-1}\right)$, and consequently on its dynamical mass $\left(M_{\mathrm{dyn}}<1.3 \times 10^{7} M_{\odot}\right)$.

Conclusions. The origin of the UCD cannot be constrained with certainty. The low metallicity, old age, and magnitude are consistent with a star cluster origin, whereas the extended size is consistent with an origin as the stripped nucleus of a dwarf galaxy with a initial stellar mass of a few $10^{8} M_{\odot}$.
\end{abstract}

Key words. galaxies: individual: NGC 1336 - galaxies: dwarf - galaxies: nuclei - galaxies: kinematics and dynamics

\section{Introduction}

Ultra compact dwarf galaxies (UCDs) were discovered about two decades ago (Minniti et al. 1998; Hilker et al. 1999a; Drinkwater et al. 2000) in studies of galaxy clusters. With masses of $10^{6}<M<10^{8} M_{\odot}$ (Mieske et al. 2013) and radii between 10 and $100 \mathrm{pc}$ (Drinkwater et al. 2003; Haşegan et al. 2005; Mieske et al. 2006), UCDs are amongst the densest stellar systems in the Universe. Compared to other dense systems, they occupy the region between globular clusters (GCs) and compact ellipticals (cEs) on the mass-size plane (e.g. Misgeld \& Hilker 2011; Brodie et al. 2011; Norris et al. 2014; Janz et al. 2016).

\footnotetext{
* Full Table B.1 is only available at the CDS via anonymous ftp to cdsarc.u-strasbg. fr (130.79.128.5) or via http://cdsarc. u-strasbg.fr/viz-bin/qcat?]/A+A/625/A50

$\star \star$ Based on observations collected at the ESO Paranal La Silla Observatory, Chile, Prog. ID 60.A-9192, PI Fahrion.
}

The intermediate nature of these objects is reflected in the proposed formation scenarios for UCDs: i) UCDs constitute the high-mass end of classical GCs (e.g. Mieske et al. 2002, 2004; Kissler-Patig et al. 2006), ii) are formed from the merging of star clusters in interacting galaxies (Fellhauer \& Kroupa 2002, 2005; Maraston et al. 2004), or iii) they are the remnant nuclei of tidally stripped dwarf or low-mass galaxies (Phillipps et al. 2001; Bekki et al. 2003; Drinkwater et al. 2003; Pfeffer \& Baumgardt 2013; Strader et al. 2013). While many UCDs occupy a similar parameter space as nuclear star clusters (NSCs; e.g. Walcher et al. 2005; Norris et al. 2015), there is evidence that the general UCD population contains both genuine star clusters and remnant nuclei (Brodie et al. 2011; Da Rocha et al. 2011; Janz et al. 2016). Very recently, Du et al. (2019) suggested the formation of cEs through ram-pressurestripping-induced star formation of a stripped, gas-rich dwarf galaxy that orbits in the hot corona of a massive galaxy. 
Although cEs are generally more massive and larger than UCDs, a similar formation scenario could apply to the most massive, metal-rich UCDs.

In the stripped nucleus scenario, tidal tails and extended envelopes around UCDs are expected. These are challenging to observe due to their short lifetimes; however, such features have been detected around some UCDs in the Fornax cluster (Voggel et al. 2016; Wittmann et al. 2016). In addition, the massive $\left(M=4 \times 10^{8} M_{\odot}\right)$ second nucleus of the merger galaxy NGC 7727, which probably resembles a UCD in formation, seems to be embedded in a tidal stream (Schweizer et al. 2018). If UCDs are remnant nuclei of stripped galaxies, one expects to find a massive black hole (BH) in the UCD's centre, similar to those found in some NSCs (e.g. Seth et al. 2008; Graham \& Spitler 2009; Neumayer \& Walcher 2012). The presence of the $\mathrm{BH}$ causes a rise in the central velocity dispersion of the UCD, observable with high angular resolution integral field unit (IFU) instruments supported by adaptive optics (AO). Recent studies of massive UCDs in the Virgo and Fornax clusters (Seth et al. 2014; Ahn et al. 2017, 2018; Afanasiev et al. 2018) have indeed detected signatures of central super massive BHs (SMBHs) that constitute up to $15 \%$ of the UCD's mass.

The presence of a central SMBH has also shed light on an interesting observational result from a decade ago. Based on measurements of structural parameters from photometry in combination with integrated velocity dispersion measurements, it was shown that many UCDs exhibit an elevated dynamical massto-light ratio $\left(M / L_{\mathrm{dyn}}\right)$ when compared to stellar population estimates (e.g. Haşegan et al. 2005; Mieske et al. 2008; Taylor et al. 2010). Originally, this trend prompted suggestions that there is a variation of the initial mass function (IMF) in UCDs. Both topheavy (Murray 2009; Dabringhausen et al. 2009) and bottomheavy IMFs (Mieske \& Kroupa 2008) are discussed. While a SMBH presents a different explanation for the elevated $M / L_{\mathrm{dyn}}$ for high-mass UCDs (Mieske et al. 2013), a search for SMBHs in two lower mass UCDs $\left(<10^{7} M_{\odot}\right)$ around Centaurus A yielded a non-detection (Voggel et al. 2018). Because of detection thresholds, the non-detection of a central $\mathrm{BH}$, however, does not disqualify a UCD from being a former nucleus of an accreted low-mass dwarf galaxy.

Nonetheless, this non-detection might imply that the population of high-mass UCDs (above $10^{7} M_{\odot}$ ) are dominated by tidally stripped nuclei while many lower mass UCDs are highmass star clusters. This is also supported by semi-analytical analyses of $\Lambda$ cold dark matter simulations (Pfeffer et al. 2014, 2016) that predicted the number and mass distribution of stripped nuclei in a cluster environment similar to the Virgo and Fornax clusters. As of now, the sample of identified NSC-type UCDs is biased to high stellar masses, where SMBHs can be found with high angular resolution IFU observations or the brightness of the object allows a detailed study of the star formation history (SFH; Norris et al. 2015). These high-mass UCDs typically were once the nuclei of stripped massive galaxies with initial masses $>10^{9} M_{\odot}$ (Norris \& Kannappan 2011; Seth et al. 2014; Ahn et al. 2018; Afanasiev et al. 2018). Nonetheless, the remnant nuclei of stripped dwarf galaxies $\left(M_{*} \sim 10^{8} M_{\odot}\right)$ should be found amongst the low-mass UCDs $\left(<10^{7} M_{\odot}\right)$, as for example $N$-body simulations show (Pfeffer \& Baumgardt 2013); however, this sample is contaminated by high-mass GCs.

Observationally, it is difficult to differentiate what fraction of UCDs at each mass bin are stripped nuclei or are formed as genuine (or merged) star clusters at these low masses. Generally, NSCs of dwarf galaxies have larger sizes and are more massive and brighter than typical GCs (Misgeld \& Hilker 2011;
Sandoval et al. 2015). SMBHs in UCDs would give direct evidence but are very difficult to measure in distant low-mass systems (Voggel et al. 2018). In addition, the nuclei of dwarf galaxies are more metal poor than their high-mass equivalents, as dictated by the mass-metallicity relation (Spengler et al. 2017), and thus have metallicities and colours comparable to the blue population of a GC system (Norris \& Kannappan 2011; Sandoval et al. 2015; Janz et al. 2016). An extended or chemically complex SFH could give evidence for a NSC origin (e.g. $\omega$ Cen, Hilker \& Richtler 2000); but studying SFHs of unresolved extragalactic UCDs requires deep spectroscopic observations (Norris \& Kannappan 2011) that are challenging for distant low-mass systems.

In the Milky Way (MW), stripped nuclei of dwarf galaxies can be identified more easily. The cluster $\omega$ Cen (NGC 5139), the most massive GC in the MW (Harris 1996) with a mass of $\sim 3 \times 10^{6} M_{\odot}$ (Baumgardt 2017; Baumgardt \& Hilker 2018), is often considered to be the remnant nucleus of an accreted dwarf galaxy due to the presence of multiple stellar populations (e.g. King et al. 2012) and its retrograde orbit (Majewski et al. 2000). The second most massive star cluster, M54 (NGC 6715, $M$ $1.4 \times 10^{6} M_{\odot}$, Baumgardt \& Hilker 2018), is classified as a GC of the MW (Harris 1996); however, it is located in the centre of the Sagittarius dwarf galaxy (e.g. Ibata et al. 1997; Bellazzini et al. 2008) and thus is considered to be a NSC in the process of being stripped by the MW. Both $\omega$ Cen and M 54 are metal poor $([\mathrm{Fe} / \mathrm{H}]=-1.62 \mathrm{dex}$ and $-1.58 \mathrm{dex}$, respectively $)$ and have absolute magnitudes of $M_{V} \sim-10.37 \mathrm{mag}$ and $-10.20 \mathrm{mag}$, respectively (Harris 1996).

In addition to observational challenges to identify low-mass UCDs amongst the GC population of a galaxy, it is unclear if the formation pathway of UCDs depends on the galaxy cluster environment in which they reside. Typically, UCDs are found at rather large projected distances $(>10 \mathrm{kpc})$ from a nearby giant elliptical galaxy located near the centre of a galaxy cluster or group. We might give the example of the first discovered UCD in Hilker et al. (1999a), named UCD3 in Drinkwater et al. (2000), which has a projected distance of $11 \mathrm{kpc}$ to the closest galaxy NGC 1404, a giant elliptical galaxy in the centre of the Fornax cluster. However, its radial velocity suggests that UCD3 is instead bound to NGC 1399, located $50 \mathrm{kpc}$ in projected distance from UCD3. It is not clear whether these large separations are connected to the formation of UCDs or whether they are a selection effect as it is difficult to perform a systematic search for UCDs in the high surface brightness regions near the centre of major galaxies.

In this paper we report the serendipitous discovery of a UCD in close projected distance $\left(r_{\text {proj }}=1.1 \mathrm{kpc}\right)$ to the low-mass earlytype galaxy FCC 47 (NGC 1336) in Multi Unit Spectroscopic Explorer (MUSE) adaptive optics (AO) science verification (SV) data. For the photometric analysis, we used archival Hubble Space Telescope (HST) data taken with the Advanced Camera for Surveys (ACS). A zoomed HST/ACS image in the F475W filter of FCC 47 is shown in Fig. 1. The white contours show the placing of the MUSE pointing. It is oriented such that the NSC of FCC 47 - the main target of the original study - is in the corner. With a projected distance of $780 \mathrm{kpc}$ to NGC 1399 , FCC 47 itself lies on the outskirts of the Fornax cluster, but was covered by photometric surveys of the cluster such as the HST ACS Fornax Cluster survey (ACSFCS, Jordán et al. 2007) and the Fornax Deep Survey (FDS, Iodice et al. 2016). However, the UCD in FCC 47 (hereafter FCC 47-UCD1) was not discovered in these surveys, probably because their method of selecting classical GCs missed this UCD due to its large size and extended 


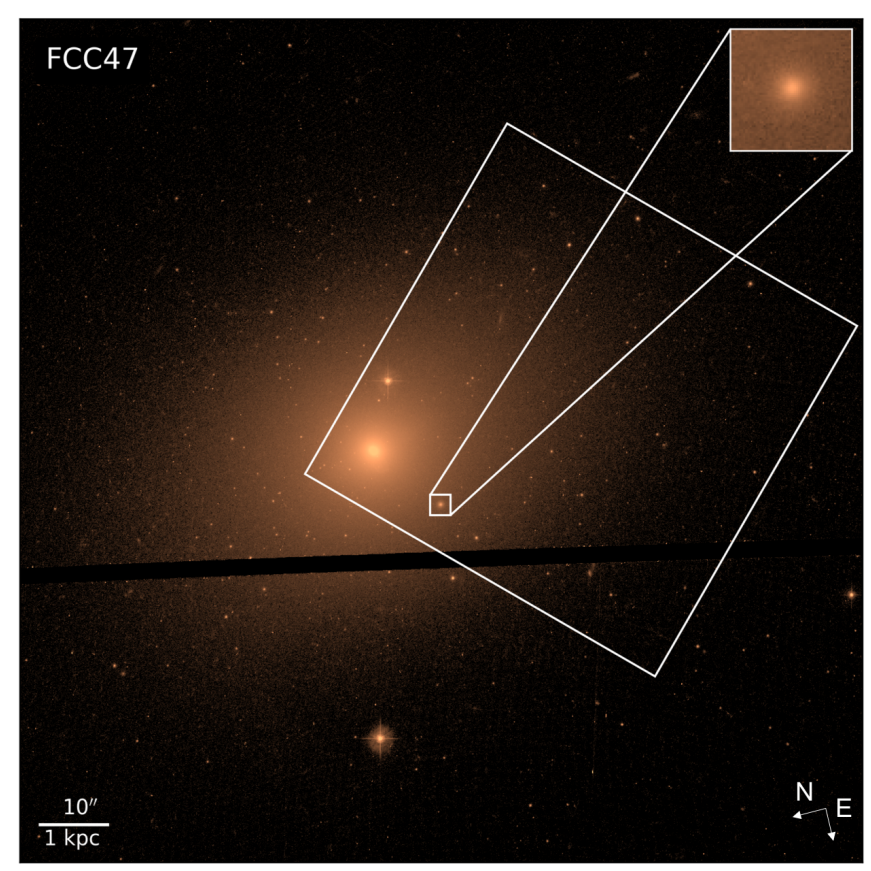

Fig. 1. Image of FCC 47 in the HST/ACS F475W filter of the central $\sim 11 \times 11 \mathrm{kpc}$. The UCD is shown in the zoomed box. The white rectangle shows the pointing of the MUSE field of view in wide field mode corresponding to $1^{\prime} \times 1^{\prime}$.

nature. Table 1 lists some basic information about FCC 47. We explore the various observational parameters of FCC 47-UCD1 to constrain the possible origin of the UCD as either a massive GC or the remnant NSC of a stripped dwarf galaxy.

This paper is organized as follows. Section 2 presents the data. Section 3 contains our structural photometric and spectroscopic analysis. The results are shown in Sect. 4 and are discussed in Sect. 5. In Sect. 6, we present a summary and the conclusions.

\section{Data}

\subsection{MUSE science verification adaptive optics data}

We discovered FCC 47-UCD1 in AO-supported IFU data of FCC 47 acquired with the MUSE instrument on the Very Large Telescope. In the wide field mode (WFM, Bacon et al. 2010) MUSE provides $\sim 90000$ spectra in an almost square $1^{\prime} \times 1^{\prime}$ field of view (FOV) with a spatial sampling of $0.2^{\prime \prime} /$ spaxel. MUSE operates in the optical wavelength regime with a mean spectral resolution of $R \sim 3000\left(\mathrm{~d} V \sim 100 \mathrm{~km} \mathrm{~s}^{-1}\right)$. Our data were acquired during the SV phase (programme 60.A-9192, P.I. Fahrion) following the commissioning of the Ground Atmospheric Layer Adaptive Corrector for Spectroscopic Imaging (GALACSI) AO system for the WFM. The data were acquired on the 17 September, 2017. The observing programme was designed to study the NSC of FCC 47 in combination with FCC 47's rich GC system (Jordán et al. 2015), which will be presented in a forthcoming paper (Fahrion et. al., in prep.). The simultaneous observation of the UCD in the MUSE FOV was purely coincidental.

The MUSE data of FCC 47 consist of ten exposures of $360 \mathrm{~s}$ each. Dedicated sky exposures of three minutes were acquired in between the on-source observations. The data were taken in the nominal mode with a wavelength coverage ranging from
Table 1. Basic information about FCC 47.

\begin{tabular}{lcc}
\hline \hline Property & Value & Reference \\
\hline RA (J2000) & $03: 26: 32.19$ & \\
Dec (J2000) & $-35: 42: 48.80$ & \\
$d(\mathrm{Mpc})$ & $18.3 \pm 0.6$ & Blakeslee et al. (2009) \\
$B_{\mathrm{T}}(\mathrm{mag})$ & 13.34 & Glass et al. (2011) \\
$r_{\text {eff }}(\operatorname{arcsec})$ & 30.0 & Ferguson (1989) \\
$M_{*}\left(M_{\odot}\right)$ & $\sim 2 \times 10^{10}$ & Fahrion et al. (in prep.) \\
\end{tabular}

Notes. ${ }^{(a)}$ Based on orbit-based dynamical model. Saulder et al. (2016) report a stellar mass of $6.4 \times 10^{9} M_{\odot}$.

4750 to $9300 \AA$. Due to the sodium lasers of the AO facility, the wavelength range between 5800 and $6000 \AA$ is filtered out. The observing conditions for FCC 47 were less than ideal with an atmospheric seeing of around 1.6". However, the GALACSI system was capable reducing this to a final point spread function (PSF) with a full width at half maximum (FWHM) of $\sim 0.7^{\prime \prime}$. Unfortunately, because there is only one bright foreground star in the FOV and it is located in the high surface brightness region near the centre of the galaxy, the data are not ideal to study the effects of the AO system on the MUSE PSF. We measured the PSF FWHM on the star with Image Reduction and Analysis Facility (IRAF) and by two-dimensional (2D) modelling with IMFIT (Erwin 2015). Both, a Gaussian model and a Moffat profile fit the PSF equally well, so for the purpose of this work we assume a Gaussian PSF with a FWHM of $\sim 0.7^{\prime \prime}$. At this image quality, the UCD is not spatially resolved in the MUSE data but is visible as a bright point source in the high surface brightness region of the galaxy.

The FCC 47 MUSE data were reduced using the MUSE data reduction pipeline version 2.2 (Weilbacher et al. 2014, 2016) incorporated in an ESO REFLEX workflow (Freudling et al. 2013). The reduction follows the steps described in Sarzi et al. (2018). It includes bias correction, flat-fielding, and a wavelength calibration. The sky subtraction was done by subtracting a model sky spectrum acquired from fitting the dedicated sky exposures. To reduce the sky residuals further, we applied the Zurich Atmosphere Purge (ZAP) algorithm version 2.0 (Soto et al. 2016). This procedure uses principle component analysis to statistically quantify and then remove the sky line residuals.

\subsection{Hubble Space Telescope/Advance Camera for Surveys data}

We complement the MUSE data with archival HST/ACS data in the F475W and F850LP filters (roughly Sloan's $g$ and $z$-bands) from the ACSFCS (PI: A. Jordán). The data are of high quality with 760 (F475W) and 1220 (F850LP) seconds of exposure time. There is also Wide Field and Planetary Camera 2 (WFPC2) data in the F606W filter (HST proposal 5446 PI: G. Illingworth) that covers FCC 47 and the UCD, but it has only 80 s of exposure time. Due to the low image quality, we do not use it for the analysis of the structural properties of the UCD in this filter, but list the integrated magnitudes in Table 2.

We processed with AsTRODRIzZLE the ACS .flc files (corrected already by the HST reduction pipeline for dark, bias, flat field, and charge transfer efficiency), and the corresponding raw files for WFPC2. Because there were only two exposures per filter, we chose to drizzle the images to their native pixel scale of the detector of $0.05^{\prime \prime} \mathrm{pix}^{-1}$ for ACS and the WFPC2 PC chip, where the UCD is located. Because FCC 47 is quite extended, 
Table 2. Photometric and structural parameters of FCC 47-UCD1.

\begin{tabular}{|c|c|c|c|c|c|c|c|}
\hline \multirow{2}{*}{$\begin{array}{c}\text { Filter } \\
(1)\end{array}$} & \multirow{2}{*}{$\begin{array}{c}m_{0} \\
(\mathrm{mag}) \\
(2)\end{array}$} & \multirow{2}{*}{$\begin{array}{c}M \\
(\mathrm{mag}) \\
(3)\end{array}$} & \multirow{2}{*}{$\begin{array}{c}\epsilon \\
(4) \\
\end{array}$} & \multicolumn{2}{|l|}{$R_{\mathrm{c}}$} & \multirow{2}{*}{$\begin{array}{l}C \\
(6) \\
\end{array}$} & \multirow{2}{*}{$\begin{array}{c}\alpha \\
(7) \\
\end{array}$} \\
\hline & & & & $\begin{array}{l}(\operatorname{arcsec}) \\
\end{array}$ & (pc) & & \\
\hline $\begin{array}{c}\text { F475W }(g) \\
\text { F606W }\end{array}$ & $\begin{array}{l}20.76 \pm 0.01 \\
20.14 \pm 0.10\end{array}$ & $\begin{array}{l}-10.55 \pm 0.01 \\
-11.26 \pm 0.10\end{array}$ & $0.08 \pm 0.01$ & $0.129 \pm 0.002$ & $11.4 \pm 0.2$ & $12.23 \pm 0.47$ & $1.69 \pm 0.07$ \\
\hline F850LP (z) & $19.30 \pm 0.01$ & $-12.01 \pm 0.01$ & $0.08 \pm 0.01$ & $0.124 \pm 0.002$ & $11.0 \pm 0.2$ & $8.96 \pm 0.34$ & $1.37 \pm 0.10$ \\
\hline
\end{tabular}

Notes. (1) HST filter, (2), (3) extinction-corrected apparent and absolute King model magnitude in the Vega system. We use $A_{\mathrm{F} 475 \mathrm{~W}}=0.04$, $A_{\mathrm{F} 606 \mathrm{~W}}=0.03$, and $A_{\mathrm{F} 475 \mathrm{~W}}=0.01$. (4) ellipticity, (5) core radius of the King model, (6) concentration parameter as the ratio between tidal and core radius, (7) exponent $\alpha$ of the King model. The uncertainties were obtained from bootstrapping with IMFIT (see Fig. A.2). The effective radii in 2D and 3D as obtained from our mass modelling method are listed in Table 3.

we disabled ASTRODRIZZLE from automatically obtaining sky levels. Instead we derived our own sky values in a region without galaxy contribution and supplied them as input. The spatially variable PSF model for each ACS filter was built using around ten foreground stars. Due to the small FOV of the WFPC2 PCchip, there were no suitable stars from which to build a PSF. Therefore, we generated the PSF with TINYTIM for the location of the UCD on the WFPC2/PC chip and drizzled the PSF images with the same ASTRODRIZZLE parameters as for the science data. For the computation of magnitudes, we used photometric zero points in the Vega system of 26.172,22.850, and 24.352 mag for the F475W, F606W, and F850LP filters, respectively.

\section{Analysis}

\subsection{Structure and photometry}

We study the photometry of FCC 47-UCD1 in the HST filters F475W $(g)$ and F850LP $(z)$. As the UCD is close in projection $\left(13^{\prime \prime}=1.1 \mathrm{kpc}\right)$ to the centre of FCC 47 , the galaxy background has to be removed. This is achieved by creating an IRAF ELLIPSE model of the host galaxy in each filter and subtracting it from the data. We find that the residual is flat at the UCD's position. The residual varies with a standard deviation of 6.4 counts $\mathrm{s}^{-1}$ around a mean flux level of 3.7 counts $\mathrm{s}^{-1}$ in the $\mathrm{F} 475 \mathrm{~W}$ filter, whereas the UCD reaches a peak of 1245 counts $\mathrm{s}^{-1}$, corresponding to a magnitude fluctuation of $\sim 0.005 \mathrm{mag}$. The structural parameters of the UCD are then determined using IMFIT (Erwin 2015), a modular procedure to fit 2D surface brightness distributions.

A variety of $2 \mathrm{D}$ surface brightness models that can be added freely with IMFIT to create multi-component or off-centre component models. In the past, UCD surface brightness profiles have been successfully fitted by either King or Sérsic profiles (e.g. Haşegan et al. 2005; Evstigneeva et al. 2007a; Mieske et al. 2008), often also using two-component models representing a dense core and a more diffuse stellar envelope. We used IMFIT to fit FCC 47-UCD1 with two single-component models (King and Sérsic, respectively) and two two-component models (King + Exponential and King + Sérsic). We used generalized King models ${ }^{1}$ that are parametrized by a core radius $R_{\mathrm{c}}$, a concentration $C=R_{\mathrm{t}} / R_{\mathrm{c}}$ as ratio between tidal and core radius, and a variable exponent $\alpha$. We used 10× oversampled PSFs in the two filters to ensure an accurate representation of the smallest scales. IMFIT allows us to determine the best-fitting parameters with a Markov-chain-Monte-Carlo (MCMC) analysis. The MCMC

\footnotetext{
1 The generalized King profile is given by

$I(R)=I_{0}\left[\frac{1}{\left(1+\left(R / R_{\mathrm{c}}\right)^{2}\right)^{\frac{1}{\alpha}}}-\frac{1}{\left(1+\left(R_{\mathrm{t}} / R_{\mathrm{c}}\right)^{2}\right)^{\frac{1}{\alpha}}}\right]^{\alpha}$.
}

analysis of our fits shows well-behaved distributions around the best-fit parameters for the single-component fits (see Fig. A.1). For the two-component fits we see degeneracies between many parameters indicating that these models over-fit the data.

The 2D surface brightness images of the UCD and the residuals after subtracting the different IMFIT models are shown in Fig. 2. The single-component fits perform well with no obvious difference between King and Sérsic models. In contrast, the two-component models tend to overestimate the UCD flux at the centre. To further investigate the fit, we show the radial intensity profiles and the relative residuals extracted with the IRAF ELLIPSE task in Fig. 3. As seen in the 2D plot, the two-component models overestimate the flux in the centre. In the $g$-band, the difference between the King and Sérsic profiles is small, but the single King profile performs slightly better, as quantified by the residuals (right panel). Similarly, the best fit to the $z$-band data is achieved with the single King profile. In both filters, the relative residuals of the single King fits are below 3\% (corresponding to a magnitude error of $\sim 0.03 \mathrm{mag}$ ) out to three core radii. Above $\gtrsim 0.5^{\prime \prime}$ the flux drops to the background level of $\sim 25 \mathrm{mag} \operatorname{arcsec}^{-2}$ creating residuals with higher relative amplitudes.

We conclude that FCC 47-UCD1 is best described by a single generalized King profile with the parameters listed in Table 2. The formal errors of the MCMC analysis are $<1 \%$. To get realistic errors, we use IMFIT's bootstrapping analysis with 1000 iterations on the MCMC parameters. The resulting histograms can be found in Fig. A.2. The bootstrapping gives no direct uncertainty for the integrated magnitude, only for the peak intensity as a parameter of the model of $I_{0}=1778.9 \pm 19.1$ counts s$^{-1}$ in the $\mathrm{F} 475 \mathrm{~W}$ filter. This corresponds to a magnitude uncertainty of $0.01 \mathrm{mag}$. For comparison, we use IRAF's PHOT task to extract magnitudes directly from the background subtracted data. Here, we obtain uncertainties of 0.007 mag for both filters, independent of the chosen aperture size. Using the original data, these uncertainties slightly increase to $\sim 0.009$ mag because of the uneven galaxy background. Systematic effects, for example created by the galaxy background, thus are very small and we find that using a median filter for the background subtraction yields the same results. We therefore adopt a magnitude uncertainty of $0.01 \mathrm{mag}$ for the integrated magnitudes in the F475W and F850LP filters. For the WFCP2/PC F606W magnitudes, we obtain an uncertainty of $0.10 \mathrm{mag}$ due to the lower image quality. Our ELLIPSE residuals are flat on the extent of the UCD and should not introduce a magnitude error larger than $0.005 \mathrm{mag}$, but we tested other methods for the background subtraction such as using a simple median filter and found no difference in the results. For the comparative analysis we transformed the ACS/WFC F475W and the WFCP2/PC F606W magnitudes to Johnson $B$ and $V$ magnitudes using, respectively, 

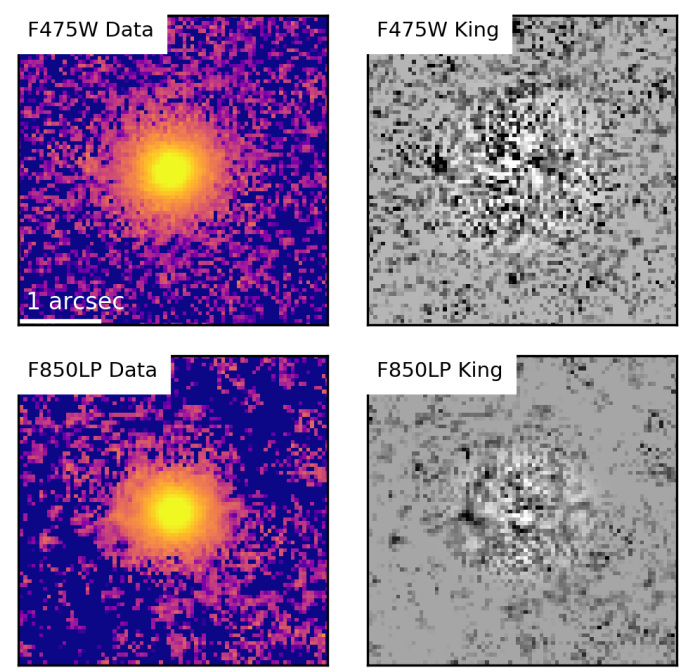
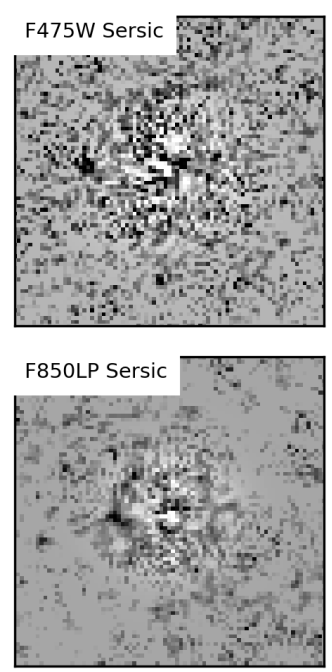
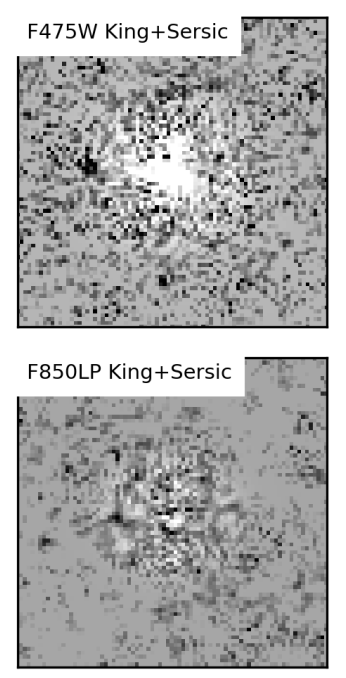

Fig. 2. Two-dimensional structural analysis. First column: HST UCD images in the F475W filter ( $g$-band, first row) and F850LP ( $z$-band, second row) after an ELLIPSE model of the galaxy background is subtracted. The second and third columns show residual images after subtracting a single generalized King model or a Sérsic model, respectively. The third and fourth columns show residuals after subtracting two-component models with a King and Sérsic and a King and exponential model, respectively. Each panel shows a $3^{\prime \prime} \times 3^{\prime \prime}(266 \times 266$ pc $)$ cut out. Black and white colours show positive and negative residuals. Figure 3 gives radial relative residuals from the various models.

the Sirianni et al. (2005) and Dolphin (2009) transformations. We obtain $V=20.37 \pm 0.10 \mathrm{mag}$ and $B=21.09 \pm 0.10 \mathrm{mag}$, corresponding to $M_{V}=-11.15 \pm 0.10 \mathrm{mag}$ using a distance of 18.3 Mpc (Blakeslee et al. 2009). The uncertainties are dominated by the photometric uncertainty of the F606W magnitude.

\subsection{MUSE spectrum}

In the MUSE data, the UCD is not resolved, but it is clearly visible as a bright point source in the FOV. While it was overlooked in the HST/ACS study of GC candidates (Jordán et al. 2015) probably due to its extended size, we can use the MUSE spectrum to confirm its membership to the FCC 47 system and can further analyse the integrated kinematics and stellar population properties.

We extracted the MUSE spectrum of the UCD in a circular aperture with a ten pixel radius and applied PSF weighting with a FWHM of $0.7^{\prime \prime}$ and a Gaussian shape. The PSF weighting is used to maximize the contribution from the UCD to the spectrum while simultaneously minimizing the influence of the strong underlying galaxy background. Since this spectrum is still heavily contaminated by the light from FCC 47's stellar body, we extracted the spectrum of the local galaxy background in an annular aperture placed around the UCD with a five pixel width and an eight pixel radius from the UCD. The background-subtracted UCD spectrum has an average signal-tonoise ratio $(\mathrm{S} / \mathrm{N})$ of $\sim 20 \AA^{-1}$ measured in a continuum region around $6500 \AA$. This $\mathrm{S} / \mathrm{N}$ is sufficient to safely derive the radial velocity $v_{\text {rad }}$ and, in principle, sufficient to determine the velocity dispersion. However, for such a low-mass system as the UCD, the velocity dispersion is close to or below the MUSE instrumental resolution as we discuss below. We also obtain the mean spectroscopic metallicity of the UCD and estimate the age. The SFH or alpha-enhancement are also not accessible at this low $\mathrm{S} / \mathrm{N}$.

We used the Penalized Pixel-Fitting code PPXF (Cappellari \& Emsellem 2004; Cappellari 2017) to extract the integrated kinematics and stellar population properties such as metallicity and age from the UCD spectrum after subtracting
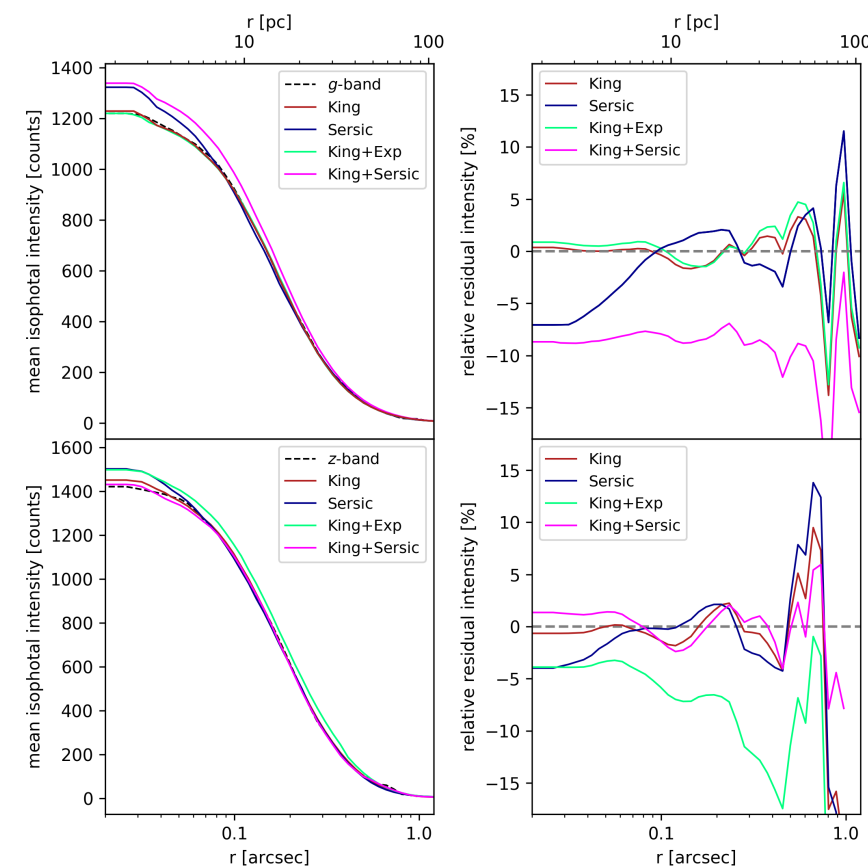

Fig. 3. Left column: UCD 1D profiles of the mean isophotal intensity versus radius in the $g$-band (first row) and $z$-band (second row) filter. The data is shown with the black dotted line, the King model fit in red, and the Sérsic model fit in blue. The two-component fits are shown in cyan (King + Exponential) and magenta (King + Sérsic). Right column: Relative residual profiles for the four different models. Above $\gtrsim 5^{\prime \prime}$ the flux drops to the background level, creating noisy radial residuals.

the galaxy contribution. PPXF is a full-spectrum fitting method that uses a penalized maximum likelihood approach to fit a spectrum with a combination of template spectra. We used the single stellar population (SSP) template spectra from the extended Medium resolution INT Library of Empirical Spectra (E-MILES, Vazdekis et al. 2012, 2016; Ricciardelli et al. 2012) because of its broad wavelength coverage. The template spectra cover a range from 1680 to $50000 \AA$, with a spectral resolution 


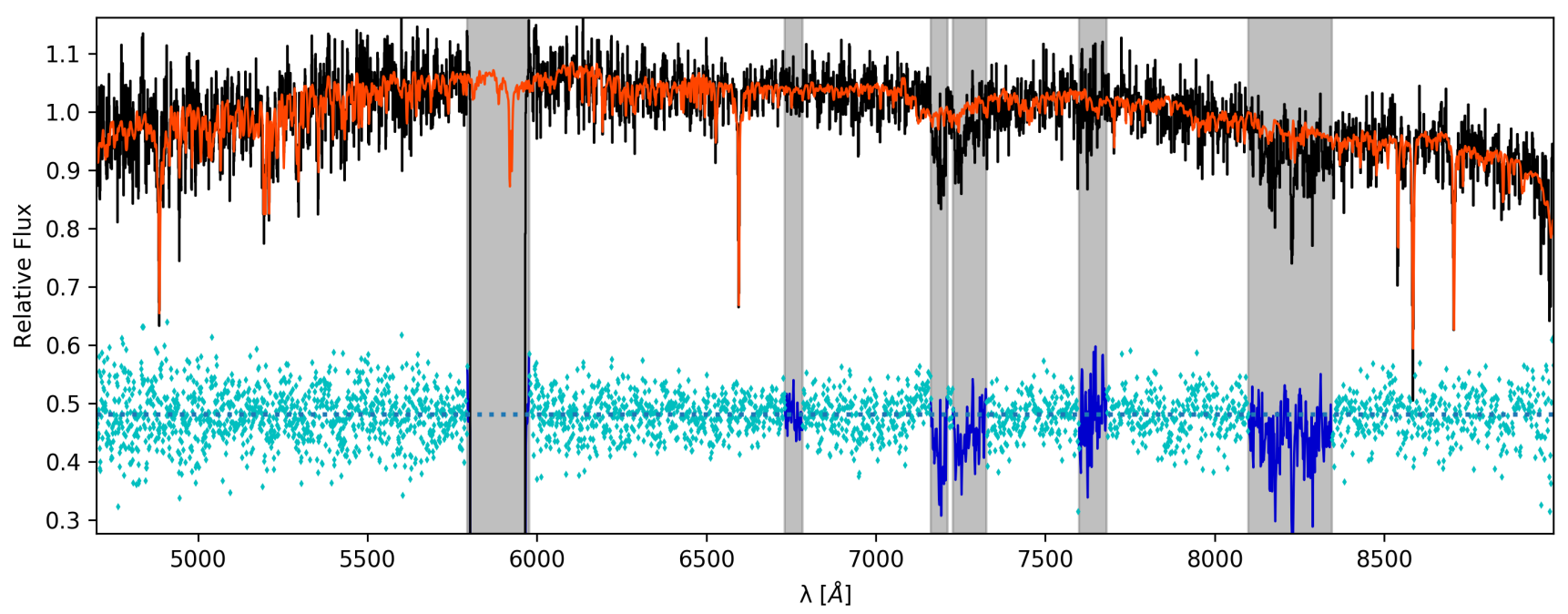

Fig. 4. $P$ PXF fit to the UCD MUSE spectrum. The original spectrum is shown in black, the PPXF fit using the E-MILES templates (Vazdekis et al. 2010) in red and the residual in blue. Masked regions with strong sky line residuals are shown in grey. The first masked region without a spectrum is due to the $\mathrm{AO}$ laser filter.

of $2.5 \AA$, the mean instrumental resolution of MUSE. We used the base $[\alpha / \mathrm{Fe}]$ models with BaSTI isochrones (Pietrinferni et al. 2004, 2006) and a double power law (bimodal) IMF with a high-mass slope of 1.30. The library offers 636 spectra in total, with 12 different metallicities from $[\mathrm{M} / \mathrm{H}]=-2.27$ to $0.4 \mathrm{dex}$ and 53 ages from $30 \mathrm{Myr}$ to $14 \mathrm{Gyr}$. The library does not offer alpha-enhanced models at this wavelength range, which might result in a slight overestimation of the metallicity if the UCD is alpha-enhanced.

Performing an unregularized fit with the full grid of EMILES SSP templates, we find a mean age of $13 \mathrm{Gyr}$ with no weights in models with ages below $11 \mathrm{Gyr}$ and a mean metallicity of $\sim-1.1$ dex. While this age estimate is probably rather uncertain due to the low $\mathrm{S} / \mathrm{N}$, we can exclude a dominant contribution to the UCD's spectrum from young stellar populations ( $<8 \mathrm{Gyr})$. This already shows that FCC47-UCD1 is clearly dominated by old stellar populations; however, neither with the MUSE spectrum nor the information from the $(g-z)$ colour (see Sect. 4.5) can we constrain the age further. In the following, we assume it to be $\sim 13 \mathrm{Gyr}$.

We determined the uncertainties on $v_{\text {rad }}$ and the mean metallicity $[\mathrm{M} / \mathrm{H}]$ in a Monte Carlo (MC)-like approach. We performed a first fit and then created 600 realisations of the spectrum by adding the residual from the first fit in a random fashion to the best-fitting model. The perturbed spectrum was then fitted again. This way, we obtain a well-sampled distribution. In each fit, we first determined the kinematics with additive polynomials of degree 10 and then fitted for the metallicity with multiplicative polynomials of degree 10 while leaving the kinematic parameters fixed. This way, we ensure that no additive polynomials are used in the stellar population fit that might change the line strengths. To avoid the effects of any age-metallicity degeneracy and to speed up the MC-runs, we restricted the template library to ages $>8$ Gyr. Figure 4 shows the original UCD spectrum with the best-fitting model overplotted in red. We show the distributions from the $600 \mathrm{MC}$ runs in Fig. A.3.

Being a low-mass system, fitting the UCD's velocity dispersion from the background-subtracted spectrum is challenging due to the low $\mathrm{S} / \mathrm{N}$ and the limited spectral resolution of the MUSE instrument. As the instrumental resolution is highest around the Calcium II triplet (CaT) at $\sim 8500 \AA$ (Guérou et al. 2017), we determined the velocity dispersion in a narrow wavelength range from 8420 to $8800 \AA$. For this fit, we used the Calcium II Triplet library (Cenarro et al. 2001), consisting of $\sim 700$ stars in a wavelength range between $8350-9020 \AA$ at $1.5 \AA$ spectral resolution. The higher resolution is relevant because the MUSE resolution as measured by Guérou et al. (2017) is below the resolution of the MILES templates of $2.5 \AA$ in the CaT region. This would require us to degrade the MUSE spectrum in this wavelength range and would further complicate the velocity dispersion measurement. We therefore chose the CaT library, selecting F, G, K, and M stars as representatives for old populations from the library and created 1000 realization as described above. This high number is needed because nearly half of all fits hit the lower limit given by PPXF. Removing these cases, we get the distribution shown in the right panel of Fig. 5. The left panel shows the MUSE spectrum around the CaT region and the PPXF fit.

\section{Results}

\subsection{Colour profile}

The surface brightness profiles of the UCD are shown in Fig. 6 together with its $(g-z)$ colour profile. The shaded areas refer to the errors from ELLIPSE. The total colour in Vega magnitudes is $(g-z)=1.46 \mathrm{mag}$. The PSF surface brightness profiles shown as dotted lines fall faster than the data and should not affect the colour profile above the core radius $\left(r \gtrsim 0.1^{\prime \prime} \simeq 2 \times F W H M_{\mathrm{PSF}}\right)$, where the PSFs are already $2.5 \mathrm{mag} \operatorname{arcsec}^{-2}$ fainter than the UCD. The PSF profiles shown in this figure are taken from the $10 \times$ oversampled PSF models. In the region, where we can exclude PSF effects and noise due to the low surface brightness in the outer parts $\left(0.1<r<0.3^{\prime \prime}\right)$, the colour profile as extracted directly from the data of the UCD shows a mild colour gradient of 0.17 mag. Using the generalized King model colour profile or as a comparison the single-component Sersic model profile, the gradient is more prominent $(0.4 \mathrm{mag})$ in this region. With our combined photometric uncertainties of $0.014 \mathrm{mag}$, this gradient is significant.

We also consider the influence of the galaxy at this position. FCC 47 is much redder than the UCD and has a colour of $(g-z)$ $\approx 2$ mag at the UCD's position. This could theoretically bias the UCD colour profile; however, the galaxy surface brightness at 

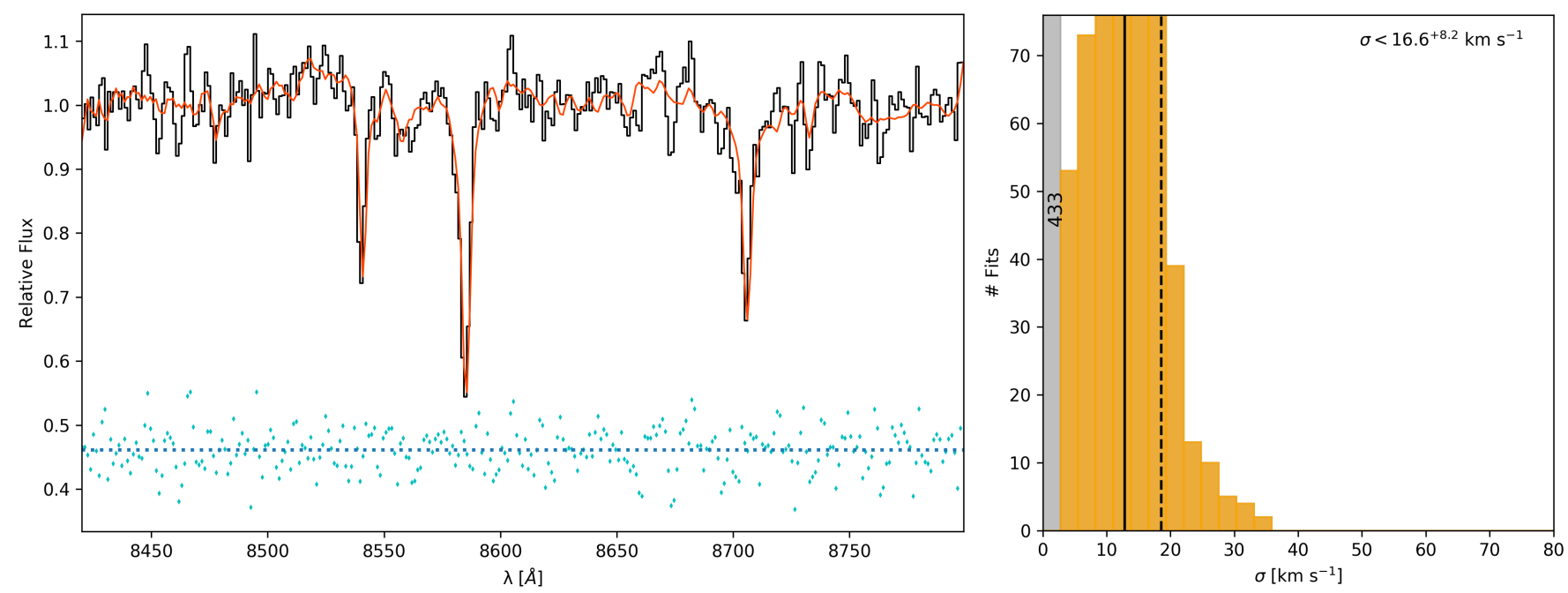

Fig. 5. Left: Ca Triplet region of the MUSE spectrum. The PPXF fit using the Ca Triplet library shown in red. Right: distributions of velocity dispersions using a MC approach with 1000 realizations of the spectrum. The 433 fits that returned zero are shown in the grey bar. The solid line gives the mean of the distribution after removing the runs that returned zero. The dotted line gives the upper $1 \sigma$ deviation.

that location is two magnitudes fainter than that of the UCD. It should therefore only account for $\sim 16 \%$ of the flux, if we did not subtract the ELLIPSE model. As our ELLIPSE residual introduces an error below $0.01 \mathrm{mag}$, we are confident that the UCD colour profile is not affected by the galaxy background.

\subsection{Comparison to GCs and Fornax and Virgo NSCs}

We show the UCD in a colour-magnitude diagram in the upper panel of Fig. 7 in comparison to the GC candidates from the ACSFCS as well as NSCs in early-type hosts in the Virgo and Fornax clusters (Côté et al. 2006; Turner et al. 2012). All magnitudes are converted to absolute Vega magnitudes using the distance moduli of $(m-M)_{\text {Fornax }}=31.51$ and $(m-M)_{\text {Virgo }}=31.09$ (Blakeslee et al. 2009). The UCD is marked by the star symbol. It is about 0.5 mag brighter than the next brightest GC around FCC 47, which is also redder. The next brightest GC with a similar colour has a magnitude difference of $\sim 1.0 \mathrm{mag}$. It is three magnitudes fainter than the NSC of FCC 47. With its magnitude and colour, the UCD sits at the bright end of the blue GC distribution and lies amongst the NSCs, especially amongst the fainter Fornax NSC population. It is bluer than many Fornax NSCs and brighter than Virgo NSCs of a similar colour. NSCs with similar $g$-band magnitudes and colours all belong to dwarf elliptical galaxies in the Fornax cluster with typical total $B$-band magnitudes of $\sim 15.5 \mathrm{mag}$ (Ferguson 1989). The lower panel of Fig. 7 shows the effective radii of GCs, NSCs, and the UCD in comparison to the $(g-z)$ colour. The UCD is more than twice as large as any GC of FCC47 and larger than most of the NSCs.

\subsection{Comparison to the globular cluster luminosity function}

As richer GC systems are more likely to form more luminous GCs (Hilker 2009; Norris \& Kannappan 2011), we evaluate whether the UCDs' magnitude is consistent with FCC 47's GC luminosity function (GCLF). The GCLF of the ACSFCS galaxies was studied by Villegas et al. (2010) using the GC candidates of all ACSFCS galaxies that were extracted following the pipeline described in Jordán et al. (2004, 2009) and were later published as catalogues by Jordán et al. (2015). All GC candidates that have a probability parameter pGC of being a GC with
pGC $>0.5$ are used. There are 276 candidates in FCC 47 and their GCLF is described by a Gaussian function with a mean magnitude of $\mu=(23.993 \pm 0.068)$ mag and a standard deviation of $\sigma=(0.988 \pm 0.053) \mathrm{mag}$ (in $A B$ magnitudes) in the $\mathrm{F} 475 \mathrm{~W}$ $g$-band. We used this GCLF to randomly draw a total number of GCs $\left(N_{\mathrm{GC}}\right)$ to create a mock GC system of FCC 47 . This was repeated 10000 times and we then counted all trails where there was at least one mock GC with a magnitude of the UCD or brighter. The resulting probability $p$ of finding at least one such GC is dependent on $N_{\mathrm{GC}}$, the total number of GCs (see Fig. 8). Harris et al. (2013) give $N_{\mathrm{GC}}=276 \pm 100 \mathrm{GCs}$, so we assume that FCC 47 does not have more than $400 \mathrm{GCs}$, probably much less. With $N_{\mathrm{GC}}=276$, the probability $p$ to find a GC as bright as the UCD is about $13 \%$. For comparison, the brightest GC $(g \sim 21 \mathrm{mag})$ from the ACS catalogue has a probability of $\sim 30 \%$, but the probability to find at least two GCs with $g<21 \mathrm{mag}$ is $\sim 7 \%$. The UCD's magnitude is therefore consistent with FCC 47's GCLF.

\subsection{Kinematics and mass estimates}

The PPXF fit to the background-subtracted MUSE spectrum reveals the radial velocity of the UCD. The UCD has a heliocentric radial velocity of $v_{\text {rad }}=(1509.7 \pm 4.5) \mathrm{km} \mathrm{s}^{-1}$. The uncertainty refers to the standard deviation from the distribution in Fig. A.3 as obtained from $600 \mathrm{MC}$ runs. The UCD has a relative velocity of $(65.3 \pm 4.9) \mathrm{km} \mathrm{s}^{-1}$ compared to the systemic velocity of FCC 47 (1444.4 $\pm 2.0 \mathrm{~km} \mathrm{~s}^{-1}$, Fahrion et. al., in prep.). The relative radial velocity of the UCD reveals that it is indeed related to FCC 47 because the velocity dispersion of the stars in FCC 47 is $\sim 80 \mathrm{~km} \mathrm{~s}^{-1}$ at the position of the UCD (Fahrion et. al., in prep.). This suggests that the escape velocity is much larger.

As described above, we determine the upper limit of the velocity dispersion using the Calcium triplet (CaT) region of the background-subtracted MUSE spectrum. From the distribution seen in Fig. 5, we find an upper limit of the velocity dispersion of $\sigma<16.6^{+8.2} \mathrm{~km} \mathrm{~s}^{-1}$. This result was obtained under the assumption that the MUSE instrumental resolution behaves as found by Guérou et al. (2017). We also test how the instrumental resolution affects the result by repeating the fits assuming a different instrumental resolution between 2.5 and $2.9 \AA$. These 


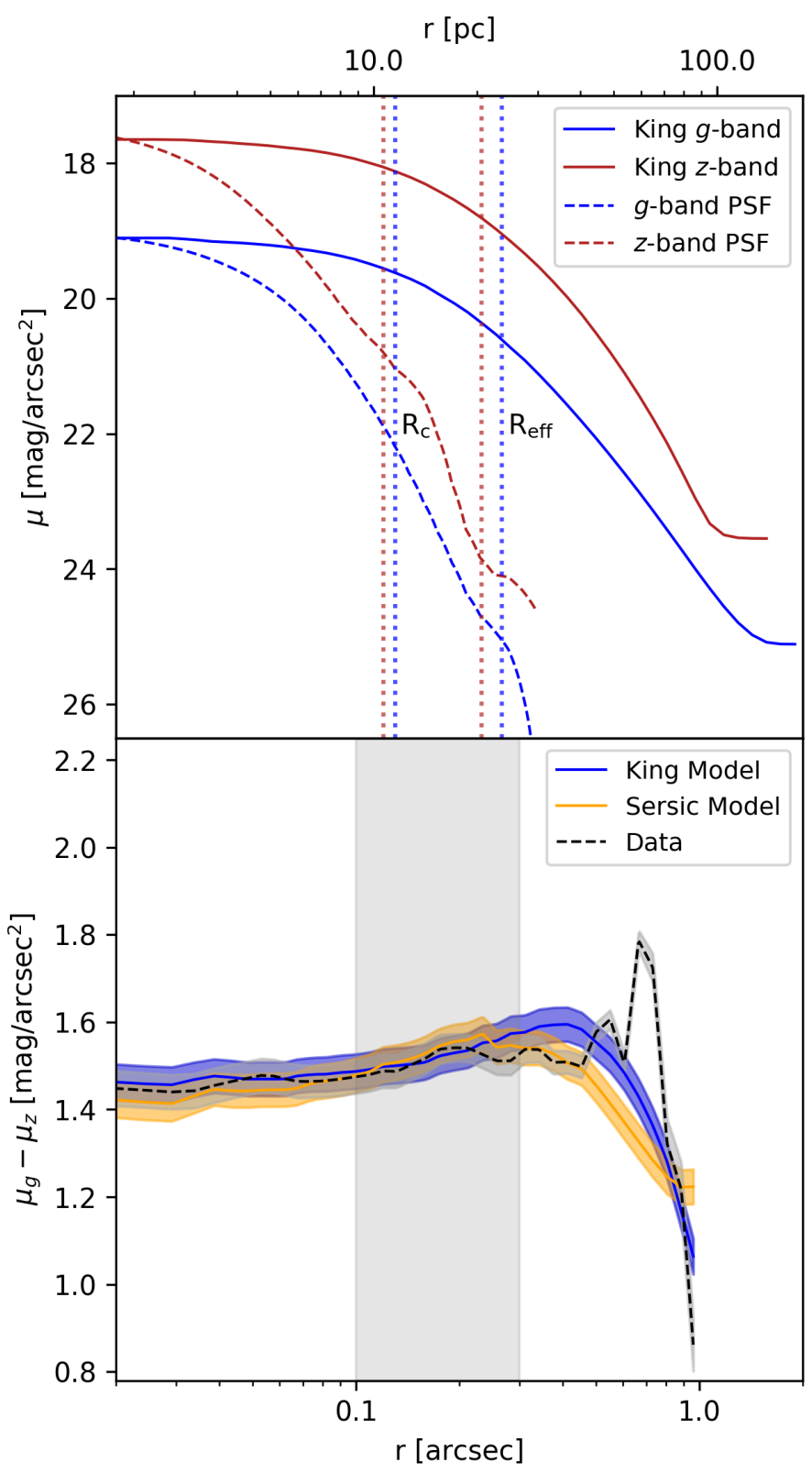

Fig. 6. Surface brightness and colour profiles. Top: UCD model surface brightness profiles for the generalized King models. We also show the profiles from the $10 \times$ oversampled PSFs shifted to match the respective filter at the centre. The vertical lines give the positions of the core and effective radii in the two filters. Bottom: $\mu_{g}-\mu_{z}$ colour profile. The blue line represents the King model colour profile, the black dotted line the data. For comparison, we also show the colour profile from the singlecomponent Sersic model. The shaded areas show the $1 \sigma$ uncertainties from the ELLIPSE extraction and the grey shaded area shows the range we use to fit the gradient.

tests give no significantly different results, partly because of the large uncertainties.

We used the mass modelling procedure described in Hilker et al. (2007) to derive an upper limit on the dynamical mass of FCC 47-UCD1. This approach uses the structural parameters as determined by the photometric fit of the generalized King profile and translates the $2 \mathrm{D}$ luminosity profile into a $3 \mathrm{D}$ density profile. This allows us to determine the value of the $2 \mathrm{D}$ and $3 \mathrm{D}$ effective radii of the UCD based on the best-fit generalized King model parameters. These values are independent from the true velocity dispersion of the UCD. We determined

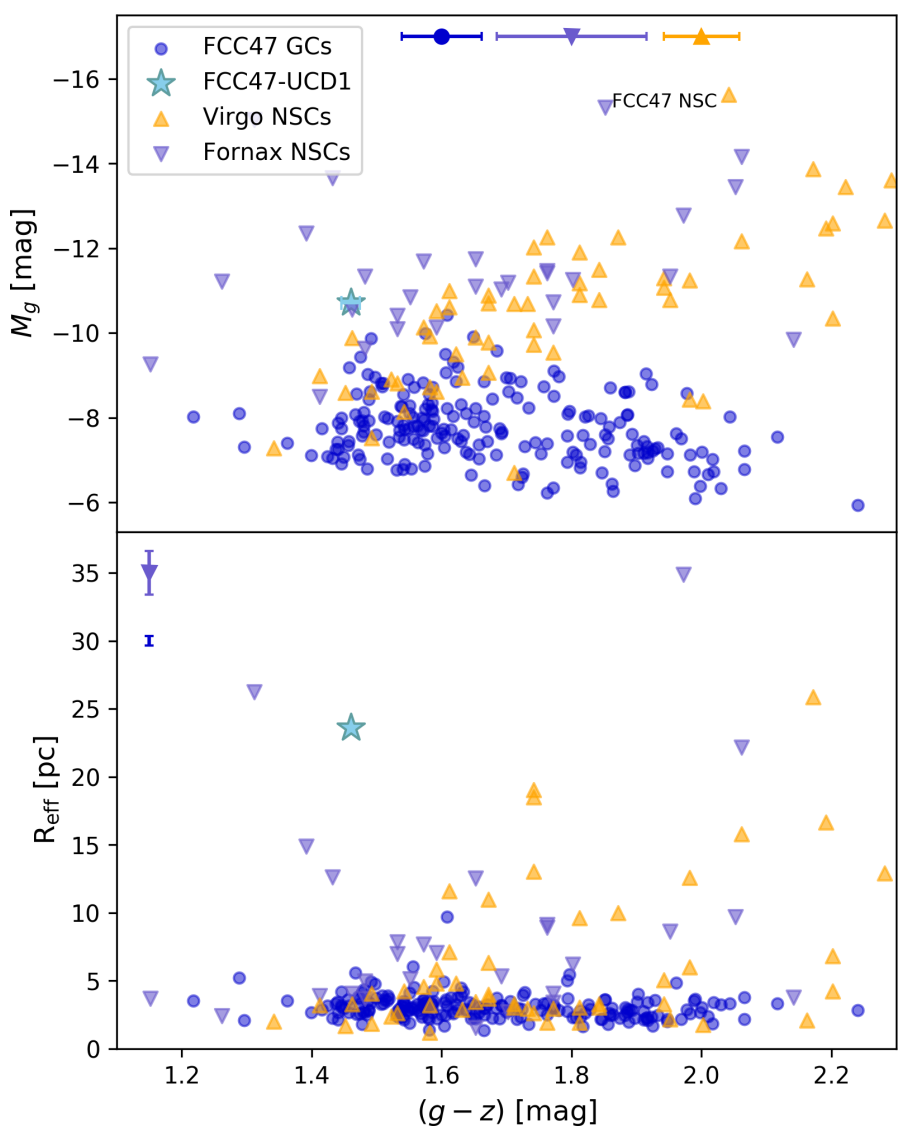

Fig. 7. Top: colour-magnitude diagram. Absolute $M_{g}$ magnitude versus $(g-z)$ colours for the UCD (light blue), the GCs in FCC 47 (blue circles, Jordán et al. 2015), and NSCs in the Virgo cluster (orange triangles, Côté et al. 2006) and in the Fornax cluster (purple triangles, Turner et al. 2012). All magnitudes are in the Vega system. Typical colour errors are shown with the symbols at the top. The uncertainty of the UCD's colour is roughly the extent of its symbol. Bottom: effective radii for GCs, NSCs, and the UCD. Typical uncertainties given at the left-hand side. There are no uncertainties given for the Virgo NSCs; however, we assume that they are comparable to the Fornax NSC uncertainties.

the uncertainty on the effective radii by combining the parameters of the generalized King models within their uncertainties to generate the lowest and highest values possible. Under the assumption of a certain total mass, the energy distribution function and cumulative mass profile are inferred from the density profile and are used to create a $\mathrm{N}$-body representation of the UCD. In the last step, the observational seeing and the aperture radius are used to derive the velocity dispersion of the model as it would be observed. By comparing this value to the observed one, the input mass is iteratively adapted until we find a match to the upper limit of the velocity dispersion. We used $N$-body representations of the UCD in both filters, each time using 100000 particles. We list the parameters inferred from this modelling in Table 3.

The modelling gives an upper limit of the dynamical mass of $M_{\text {dyn }}<1.3^{+1.6} \times 10^{7} M_{\odot}$ using the King model parameters in the F475W filter. The quoted uncertainty was estimated by not using the mean of the velocity dispersion distribution but the upper $1 \sigma$ deviation. Using the F850LP King model parameters basically gives the same result. The lower limit on the dynamical mass cannot be constrained with our spectrum; however, we can assume that it cannot be less than the photometric mass. This 


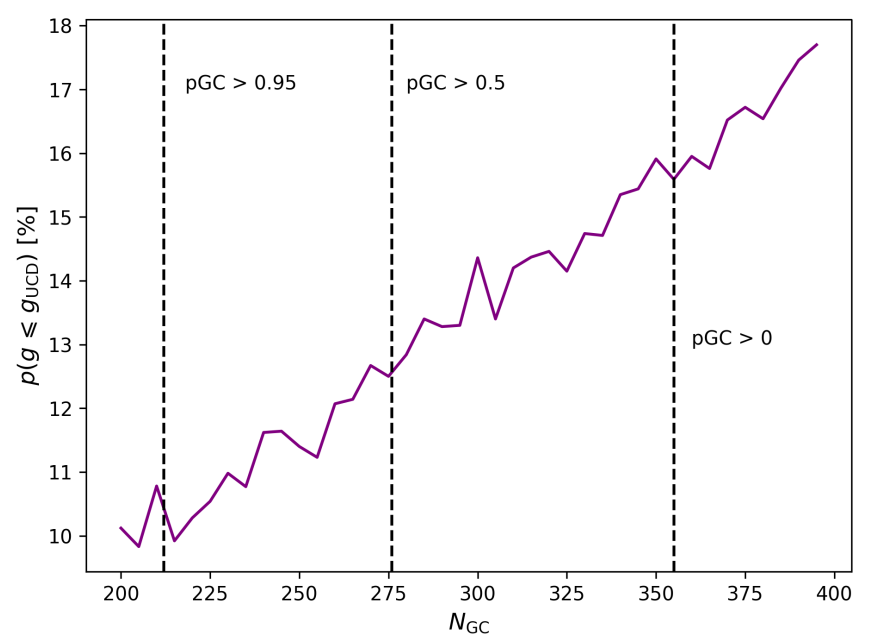

Fig. 8. Probability $p$ of finding at least one GC with a $g$-band magnitude less or equal to the UCD's magnitude versus the number of GCs $\left(N_{\mathrm{GC}}\right)$ that are drawn from FCC 47's GCLF. For each $N_{\mathrm{GC}}, 10000$ mock GC systems are drawn from the GCLF to compute the probability. The dotted lines indicate the number of GC candidates from the catalogue of Jordán et al. (2015) that have pGC parameters larger than 0, 0.5, and 0.95 , respectively.

Table 3. Parameters from the mass modelling.

\begin{tabular}{cccc}
\hline \hline Filter & $\begin{array}{c}R_{\text {eff,2D }} \\
(\mathrm{pc})\end{array}$ & $\begin{array}{c}R_{\mathrm{eff}, 3 \mathrm{D}} \\
(\mathrm{pc})\end{array}$ & $\begin{array}{c}M_{\text {dyn }} \\
\left(10^{7} M_{\odot}\right) \\
(1)\end{array}$ \\
$(2)$ & $(3)$ & $(4)$ \\
\hline F475W $(g)$ & $23.6 \pm 1.4$ & $31.5 \pm 1.9$ & $<1.30^{+1.60}$ \\
F850LP $(z)$ & $20.6 \pm 1.5$ & $27.5 \pm 2.0$ & $<1.32^{+1.60}$ \\
\hline
\end{tabular}

Notes. (1) HST filter, from the $N$-body model: (2) $2 \mathrm{D}$ and (3) $3 \mathrm{D}$ effective radius, and (4) upper limit on the dynamical mass.

upper limit is comparable to masses of other UCDs with similar effective radii (e.g. Mieske et al. 2008).

\subsection{Stellar population analysis}

In addition to the kinematics, the fit with PPXF gives an estimate of the mean metallicity. While spectroscopic metallicities are in general more reliable due to the well-known colour-metallicity degeneracy (Yoon et al. 2011), our MUSE spectrum has a rather low $\mathrm{S} / \mathrm{N}$ and the MUSE wavelength range is not ideal to accurately constrain the age of old populations, especially at low metallicities. As mentioned above, we find that the UCD is old, with a best-fitting age of $\sim 13 \mathrm{Gyr}$, and can exclude a significant contribution from a young stellar population. From fitting the spectrum with an age-restricted SSP library, we obtained the distribution in Fig. A.3 from $600 \mathrm{MC}$ runs and found a metallicity of $[\mathrm{M} / \mathrm{H}]=-1.12 \pm 0.10 \mathrm{dex}$. The uncertainty refers to the standard deviation of the distribution.

We tested the robustness of this result using different SSP templates, in particular using the so-called Padova+00 isochrones (Girardi et al. 2000) instead of BaSTI isochrones or the MILES templates on a restricted wavelength range. Performing the same number of fits gives similar results within our uncertainties. Using the photometric predictions of the E-MILES SSP models and the $(g-z)$ colour, we find a photometric metallicity of $\sim-1.4$ dex assuming an age of $13 \mathrm{Gyr}$. Assuming a younger age between 10 and 12 Gyr results in a better match with the spectroscopic metallicity but due to the uncertainty on the metallicity it is not possible to further constrain the age using the colour. Nonetheless, the comparison to photometric predictions confirms that FCC47-UCD1 is old ( $>8 \mathrm{Gyr}$ ) and clearly dominated by a metal-poor population.

We translate the metallicity into a photometric $M / L$ using the E-MILES SSP predictions. Unfortunately, the SSP predictions for $M / L$ do not cover the F850LP filter, but we can use the $M / L$ prediction for the $\mathrm{F} 475 \mathrm{~W}$ filter. We interpolate the given grid of SSP models to draw 5000 values of the $M / L$ ratio using a Gaussian distribution of the metallicity with a mean of -1.12 dex and standard deviation of 0.10 dex. As before, we assume an age of $13 \mathrm{Gyr}$. We find $M / L_{\mathrm{SSP}, g}=2.42_{-0.10}^{+0.08} \frac{M_{\odot}}{L_{\odot}}$ and with a total luminosity of the UCD of $L_{\mathrm{UCD}}=2.01 \times 10^{6} L_{\odot}$ based on its $g$-band magnitude and a distance of $18.3 \mathrm{Mpc}$, this translates to a photometric stellar mass of $M_{*}^{g}=4.87_{-0.16}^{+0.21} \times 10^{6} M_{\odot}$. This is roughly half of the upper limit we derived for the dynamical mass. While $M / L_{\mathrm{SSP}}=2.42$, we find an upper limit of $M / L_{\text {dyn }}<$ 6.47 .

\section{Discussion}

\subsection{UCD properties}

In the following, we will discuss the properties of FCC 47-UCD1 with respect to both UCD formation scenarios: formation as a genuine star cluster and as the remnant NSC of a stripped galaxy. In previous studies, the light profiles of many massive UCDs $\left(M>10^{7} M_{\odot}\right.$ ) have been found to be best represented by a dense central component together with a more extended diffuse halo (Evstigneeva et al. 2008; Strader et al. 2013; Ahn et al. 2018; Afanasiev et al. 2018). With respect to simulations by Pfeffer \& Baumgardt (2013), this two-component structure is in agreement with the stripped nucleus formation scenario, where the central component is interpreted as the remnant NSC of the UCD progenitor while the diffuse component is interpreted as the remains of the stripped galaxy. In the case of the FCC 47UCD1, we find that the structure is best represented by a single generalized King model. We do not detect a second component that could represent a stellar envelope. This is consistent with FCC 47-UCD1 being a high-mass star cluster with no extended envelope and a large effective radius of $24 \mathrm{pc}$, but a small envelope might also be hidden by our resolution limit and might cause the large measured effective radius. Pfeffer \& Baumgardt (2013) explored the formation of UCDs via tidal stripping of nucleated dwarf galaxies with simulations and found that extended envelopes fall below our surface brightness limit of 25 mag $\operatorname{arcsec}^{-2}$ already $2 \mathrm{Gyr}$ after the stripping.

FCC 47-UCD1 has a $g$-band magnitude of $g=20.76 \mathrm{mag}$ and is therefore $\sim 0.5$ mag brighter than the brightest GC from the ACSFCS sample (Jordán et al. 2015). As our consideration of the GCLF shows, the probability finding a GC with this magnitude from FCC 47's GCLF is $\sim 13 \%$. The UCD magnitude is therefore still consistent with the GCLF. With this magnitude, FCC 47-UCD1 is fainter than the previously confirmed NSCtype GCs.

The core radius obtained from the generalized King models of $\sim 11 \mathrm{pc}$ is larger than what was found for many other UCDs in Fornax and Virgo studied by Mieske et al. (2008), but we find comparable values for the $\alpha$ parameter that give the slope of the density profile and the effective radius of $24 \mathrm{pc}$ as also similar to other UCDs. For a more thorough comparison, we put the UCD in mass-size and mass-surface mass density plots in Fig. 9 similar to those presented for example in Misgeld \& Hilker (2011), Norris et al. (2014) and Sandoval et al. (2015). The 


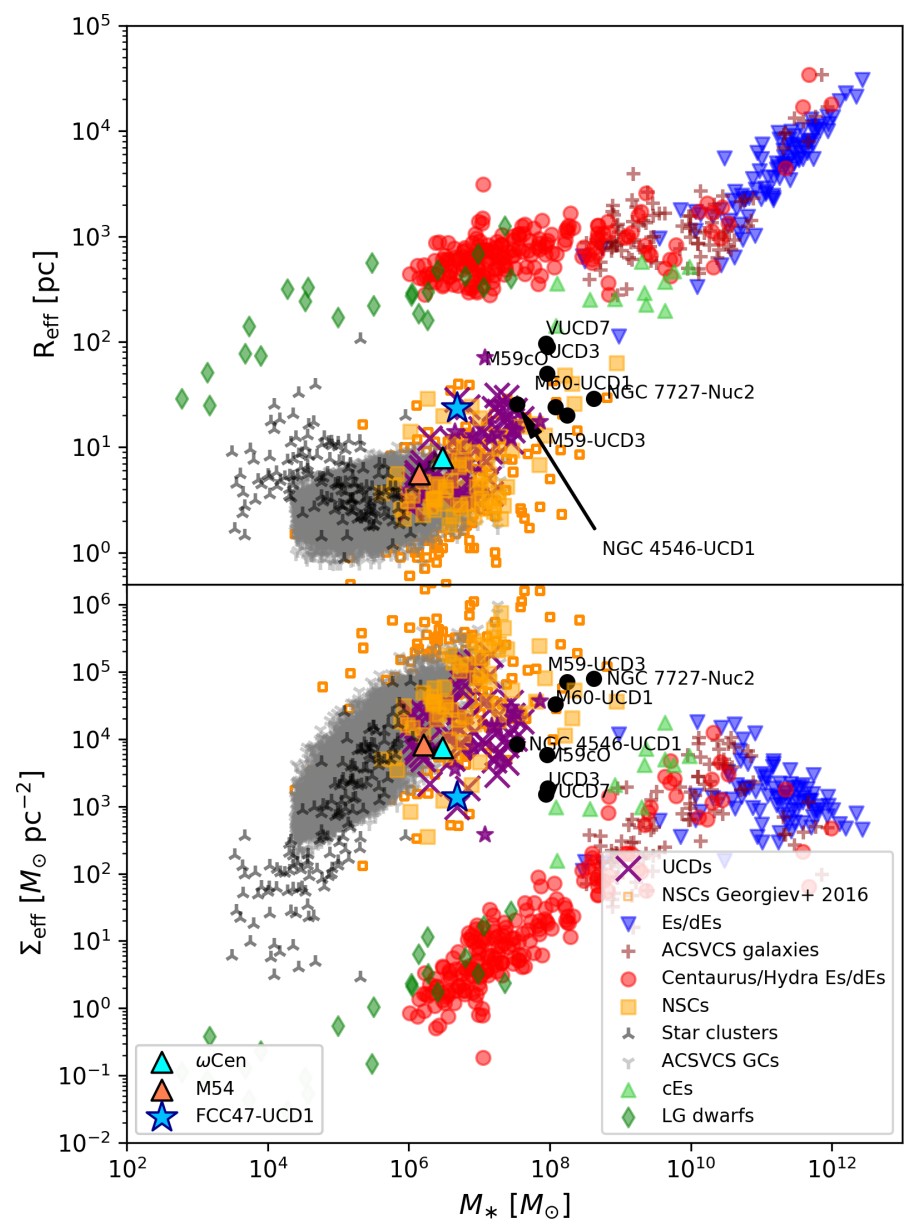

Fig. 9. Top: effective radii of stellar systems versus stellar mass. Bottom effective surface mass density $\left(\Sigma_{\text {eff }}=M_{*} / 2 \pi R_{\text {eff }}\right)$ versus stellar mass. The data were mostly collected and presented in Misgeld \& Hilker (2011). We include a few more UCDs from Norris \& Kannappan (2011), Liu et al. (2015), Sandoval et al. (2015) and Schweizer et al. (2018) and NSCs from Georgiev et al. (2016). Known NSC-type UCDs are marked by the solid black dots as well as M54 and $\omega$ Centauri. FCC 47-UCD1 is given by the blue star.

collected data is mostly taken from Misgeld \& Hilker (2011) and references therein, but we also include a few UCDs from Liu et al. (2015), Norris \& Kannappan (2011), Schweizer et al. (2018) with available mass measurements. Known NSC-type UCDs are marked as well as M54 and $\omega$ Cen (Harris 1996; Baumgardt 2017). The latter are officially classified as GCs of the Milky Way, but most likely are the stripped nuclei of accreted dwarf galaxies (e.g. Zinnecker et al. 1988; Ibata et al. 1997; Hilker \& Richtler 2000; King et al. 2012; Bellazzini et al. 2008). FCC 47-UCD1 is larger than similar bright GCs and larger than most of the shown NSCs and UCDs. Its size is comparable to some diffuse star clusters, but these have much lower masses (e.g. Liu et al. 2016). The comparison to known NSC-type UCDs shows that these are more massive, bigger, and denser than FCC 47-UCD1.

As UCDs are considered to be unrelaxed systems (Fellhauer \& Kroupa 2002; Norris \& Kannappan 2011; Voggel et al. 2018), a colour gradient translates directly into a population gradient (instead of being an effect of mass segregation), where a slightly younger population is found at the centre while the outskirts are dominated by a older, metal-poor population. Although we cannot find a significant colour gradient, we find the UCD to appear larger in the blue than the red filter by $\sim 10 \%$, corresponding to a more extended metal-poor population. As FCC 47-UCD1 is unresolved in the MUSE data, we cannot study a possible gradient spectroscopically. Our PPXF fit gives no indication of multiple populations, possibly because the $\mathrm{S} / \mathrm{N}$ is insufficient to disentangle multiple populations with this small colour difference.

The MUSE spectrum gives a mean metallicity of $[\mathrm{M} / \mathrm{H}]=-1.12 \mathrm{dex}$, which is more metal poor than what was found for most massive UCDs (e.g. Mieske et al. 2013; Janz et al. 2016; Seth et al. 2014; Ahn et al. 2018) In particular, FCC 47-UCD1 is clearly more metal poor than the confirmed NSC-type UCDs that are typically characterized by elevated metallicites (e.g. Janz et al. 2016). However, there are other UCDs with similarly low metallicities, such as NGC 3923-UCD2 and UCD3 or S999 (Norris \& Kannappan 2011; Janz et al. 2016). The metallicity is comparable to some of the GCs found in FCC 47 as will be presented in Fahrion et al. (in prep.) while other GCs have a higher metallicity. The low metallicity and old age of the UCD is consistent with it being a genuine star cluster similar to the blue GCs found in FCC 47; however, many NSCs of dwarf galaxies are known to have a similar low metallicity (Paudel et al. 2011; Sandoval et al. 2015).

The upper limit of the ratio of dynamical-to-photometric $M / L \Gamma<2.68$ is above the average value of 1.7 (Mieske et al. 2013) but well within the scatter, and the true value could be well below. If higher spectral resolution observations found an elevated dynamical $M / L$, this could indicate the presence of a central $\mathrm{BH}$ as found in other, more massive UCDs (Mieske et al. 2013; Seth et al. 2014). Alternatively, it could be attributed to IMF variations (Mieske \& Kroupa 2008; Mieske et al. 2013) or tidal stripping (Forbes et al. 2014; Pfeffer \& Baumgardt 2013). However, the low mass and apparent magnitude of FCC 47UCD1 limit the deep spectroscopic and/or photometric observations that would be needed to detect these features.

The presented photometric, kinematic, and structural properties of FCC 47 do not allow us to unambiguously pinpoint its origin. The magnitude, colour, metallicity, and the absence of an extended envelope are consistent with it being a very massive star cluster; however, the stripped NSC of a dwarf galaxy can show similar properties (see e.g. Pfeffer \& Baumgardt 2013). The very large effective radius of $\sim 24 \mathrm{pc}$ makes it a complete outlier from the rest of FCC 47's GCs. This is perhaps the most convincing argument that this object is different from typical GCs, making FCC 47-UCD1 a candidate for being the stripped nucleus of a dwarf galaxy, similar to the ones we find in the MW (e.g. $\omega$ Cen and M 54).

\subsection{Implications for the stripped nucleus scenario}

Under the assumption that FCC 47-UCD1 is the remnant nucleus of a stripped dwarf galaxy, in this section we explore the properties of its hypothetical progenitor galaxy.

\subsubsection{Comparison to Fornax NSCs}

As the colour magnitude diagram reveals (Fig. 7), the magnitude and colour of FCC 47-UCD1 is comparable to several NSCs of galaxies in the Fornax clusters. We find that the UCD has almost the same colour and magnitude as the NSC of FCC19, a nucleated dwarf elliptical galaxy with a $B$-band magnitude of 15.2 (Ferguson 1989). Assuming a $M / L_{\mathrm{B}} \sim 2$ as typical for such a galaxy (e.g. Bell \& de Jong 2001), FCC19 probably has a mass of a few $10^{8} M_{\odot}$. We find $B$-band magnitudes for the galaxies 
FCC100 and FCC202, two other nucleated dwarf galaxies that both have NSCs with a similar brightness to FCC 47-UCD1 but at a redder colour. While there is no mass estimate available for FCC 100, Eigenthaler et al. (2018) report a stellar mass of $M_{*}=1.9 \times 10^{9} M_{\odot}$ for FCC 202. Under the assumption that FCC 47-UCD1 would be the stripped nucleus of a galaxy, it is likely that its progenitor had a similar mass as FCC 19 has today. However, we cannot determine if the progenitor was an elliptical dwarf galaxy like the dwarfs we see in the Fornax cluster today or if it rather had a spiral or irregular morphology. Some dwarf irregulars are known to host massive old clusters in their nuclear regions that are separated from the normal GCs by a magnitude gap of $\sim 0.5 \mathrm{mag}$ (Georgiev et al. 2009a).

\subsubsection{Nuclear star cluster-galaxy mass relation}

It has long been established that the properties of nuclear regions of galaxies, such as the central SMBH or the NSC, scale with the properties of the galaxy itself (Magorrian et al. 1998; Ferrarese et al. 2006; Graham \& Driver 2007; Kormendy \& Ho 2013). Georgiev et al. (2016) studied the relation between stellar mass of the NSC and host stellar mass for both early- and late-type galaxies (ETGs/LTGs) with masses $>10^{8} M_{\odot}$. In case FCC 47-UCD1 indeed is of NSC-origin, we can use these relations to predict the mass of the UCD progenitor if we assume that its stellar body has been stripped entirely without altering the nucleus. Assuming an early-type progenitor, we find a progenitor mass of $M_{\star, \text { prog,ETG }}=(3.1 \pm 0.3) \times 10^{9} M_{\odot}$, and $M_{\star, \text { prog,LTG }}=(5.9 \pm 0.6) \times 10^{9} M_{\odot}$ assuming a latetype progenitor. This is around ten times larger than the typical mass of a dwarf galaxy and probably overestimates the mass of a hypothetical progenitor of FCC 47-UCD1 as the used relations were derived using giant galaxies rather than dwarfs.

Spengler et al. (2017) did a similar study using NSCs in the Virgo cluster to extend the relation of Georgiev et al. (2016) to lower NSC and host masses. The authors give no analytical expression for their relation, but find that the relation between NSC mass and host mass flattens for host masses below $10^{8} M_{\odot}$. Their relation shows a large scatter that can comprise up to two orders of magnitude for a given nucleus mass, especially for nuclei with masses $<10^{6} M_{\odot}$. For the stellar mass of the UCD, a stellar mass of the progenitor between $10^{7}$ and $10^{9} M_{\odot}$ is within the scatter.

Using nucleated dwarf galaxies observed in the Next Generation Fornax Survey, Ordenes-Briceño et al. (2018) extended the relation between nucleus mass and host mass down to host masses of $10^{6} M_{\odot}$. The authors also find a flattening of the canonical relation towards lower masses. Using this relation and the photometric mass estimate of the UCD, the estimated mass of a possible progenitor galaxy is $M_{* \text {,prog }}=(7.2 \pm 0.4) \times 10^{8} M_{\odot}$. From the presented NSC-galaxy mass relations, the last is likely the most applicable one for estimating a possible progenitor galaxy of FCC 47-UCD1 because it was derived using dwarf galaxies while the other can still give a reasonable order of magnitude estimate.

\subsubsection{Mass-metallicity relation}

It is well established that the average metal content of a galaxy is correlated with its stellar content (Lequeux et al. 1979; Tremonti et al. 2004; Gallazzi et al. 2005; Mendel et al. 2009). Studies in the Fornax and Virgo clusters have shown that
UCDs typically have high metallicities for their luminosities and lie above the canonical metallicity-luminosity trend for elliptical galaxies (Chilingarian et al. 2011; Francis et al. 2012). Instead, they seem to follow the metallicity-luminosity trend of dwarf galaxies. For dwarf galaxies with stellar masses below $\sim 10^{10} M_{\odot}$, Kirby et al. (2013) have found a tight relationship between stellar mass and iron metallicity:

$[\mathrm{Fe} / \mathrm{H}]=(-1.69 \pm 0.04)+(0.30 \pm 0.03) \log \left(\frac{M_{*}}{10^{6} M_{\odot}}\right)$

As the used E-MILES SSP models give $[\mathrm{M} / \mathrm{H}]$ values instead of direct measurements of the iron abundances, we tested the difference between the two by fitting the MUSE spectrum again with scaled solar MILES models that have $[\mathrm{M} / \mathrm{H}]=[\mathrm{Fe} / \mathrm{H}]$ with no alpha-enhancement. The original MILES SSP models have a restricted wavelength range between 3500 and $7500 \AA$, so redder wavelengths were not used in this comparison. As we find no difference in the resulting metallicity, we assume that the iron metallicity of the UCD is the same as the total metallicity within our uncertainties.

We use the relation in Eq. (1) to estimate the stellar mass of a potential UCD progenitor under the assumption that the measured UCD metallicity represents an average of the progenitor galaxy. Usually, the highest metallicities are found at the centre of galaxies (Couture \& Hardy 1988; Koleva et al. 2011), so that the UCD's metallicity would give an upper limit on the stellar mass of a possible progenitor. However, this depends on the time of the stripping as it is possible that the progenitor galaxy today might have a higher metallicity from continuing star formation if it were not stripped. This is supported by the finding that UCDs typically have a lower metallicity than NSCs of a similar mass (Paudel et al. 2011; Spengler et al. 2017), because they are dominated by star cluster-type UCDs. Confirmed NSC-type UCDs have metallicities comparable to massive NSCs. We therefore assume that the UCD metallicity is a lower limit of what the UCD progenitor would have today without stripping and consequently the resulting mass gives a lower limit. Using the spectroscopic $[\mathrm{M} / \mathrm{H}]=-1.12 \pm 0.10 \mathrm{dex}$, the estimated stellar mass of a hypothetical UCD progenitor is $M_{*, \text { prog }}=(7.94 \pm 0.24) \times 10^{7} M_{\odot}$.

Under the assumption that FCC 47-UCD1 is a stripped NSC, the presented scaling relations imply that its potential progenitor was a dwarf galaxy similar to those found today in the Fornax cluster with a mass of a few $\sim 10^{8} M_{\odot}$. In this case, the comparison to the photometric mass of $M_{\mathrm{SSP}}=4.87 \times$ $10^{6} M_{\odot}$; this implies that the UCD progenitor lost $>95 \%$ of its mass in a $\sim 1: 100$ merger with FCC 47 . Such a dwarf galaxy might have brought a few GCs with it that should now be in the halo of FCC 47 amongst the blue GC population. However, identifying those in FCC 47's rich GC system is challenging.

\subsection{Implications for star cluster origin}

The low metallicity, old age, and magnitude of FCC 47-UCD1 are consistent with it being a very massive extended star cluster. In the MW, no GCs with this mass and size are known, but they have been observed in other galaxies (e.g. in M 87 Strader et al. 2011) and Murray (2009) proposed that very massive GCs might follow a mass-size relation leading to an extended size. In this case, FCC 47-UCD1 would mark the high-mass (high luminosity) end of the blue GC population. Here, we want to explore the implications in the case that FCC 47-UCD1 is indeed a genuine star cluster. 


\subsubsection{Dynamical friction}

Ultra compact dwarfs and massive star clusters that are close to their host's centre should spiral inwards due to dynamical friction. The timescale of inspiral of a satellite on a circular orbit in a singular isothermal sphere with velocity dispersion $\sigma_{\text {sat }}$ from an initial radius $r_{i}$ to the centre of a galaxy with characteristic velocity dispersion $\sigma_{\text {gal }}$, is given by (Binney \& Tremaine 1987)

$t_{\text {fric }}=\frac{2.7 \mathrm{Gyr}}{\ln \Lambda} \frac{r_{i}}{30 \mathrm{kpc}}\left(\frac{\sigma_{\mathrm{gal}}}{300 \mathrm{kms}^{-1}}\right)^{2}\left(\frac{\sigma_{\mathrm{sat}}}{100 \mathrm{kms}^{-1}}\right)^{-3}$,

where $\Lambda$ is the Coulomb logarithm. Under the assumption that the satellite has a significantly smaller dispersion than the galaxy $\Lambda=2^{2 / 3} \sigma_{\text {gal }} / \sigma_{\mathrm{s}}$. For FCC $47, \sigma_{\text {gal }} \approx 80 \mathrm{~km} \mathrm{~s}^{-1}$, as we determine from the velocity dispersion map presented in Fahrion et al., in prep.

If we assume that FCC 47-UCD1 is on such a circular orbit around the galaxy with its $3 \mathrm{D}$ separation given by the projected distance of $1.1 \mathrm{kpc}$, the inspiral timescale to the centre is less than 0.5 Gyr. The short dynamical timescale allows different interpretations. Firstly, if the UCD is of GC origin, a near-circular orbit is plausible, implying that the UCD will soon merge into the NSC of FCC 47. In this case, it must have formed much further out on the outskirts of FCC 47 to still be observed today. Choosing an initial radius of $\sim 50 \mathrm{kpc}$, the inspiral time increases to $>9$ Gyr. Such a large initial distance could explain why FCC 47-UCD1 is still observable today, but the formation of such a massive star cluster might be difficult at large galactocentric radii due to the low gas density, even at early times (e.g. Pfeffer et al. 2018). Following the argumentation of Norris \& Kannappan (2011), who have compared the dynamical timescale of GC-type and NSC-type UCDs, long-lived (old) massive objects such as FCC 47-UCD1 are not expected to have these short dynamical timescales on average if they are of GC origin because only a few would be caught by chance right before their merger into the galaxy centre.

The dynamical friction timescale as presented here assumes a circular orbit in an isothermal sphere; however, the assumption of an isothermal sphere might not be valid because the dynamical orbit-based Schwarzschild model of FCC 47 as presented in Fahrion et al. (in prep.) shows that FCC 47 is a triaxial galaxy. This model also shows that the circular velocity at the UCD's position is around $\sim 100 \mathrm{~km} \mathrm{~s}^{-1}$, whereas the UCD has a relative velocity of $\sim 65 \mathrm{~km} \mathrm{~s}^{-1}$. This could indicate that the projected distance gives an underestimation of the true 3D separation under the assumption of a circular orbit, which in turn would also increase the dynamical friction timescale. The assumption of a circular orbit might also not apply in case FCC 47-UCD1 is of ex-situ origin. If it was located in an accreted dwarf galaxy, it is more likely that it came in on a radial orbit that has on average a much larger dynamical friction timescale.

\subsubsection{Ex-situ origin}

In the context of the hierarchical assembly of galaxies, the blue GC sub-population is often interpeted as having formed in low-mass dwarf galaxies and then been accreted during merger events (Côté et al. 1998, 2006; Hilker et al. 1999b; Lotz et al. 2004; Peng et al. 2006; Georgiev et al. 2008). Indications of such an ex-situ origin are often given by stark contrasts in chemical properties between the host galaxy and the GC or UCD. (Norris \& Kannappan 2011) and the red GC system (Côté et al. 2006). The stellar body of FCC 47 at the UCD's location is dominated by an old, gas-free population that is much more metal rich $([\mathrm{M} / \mathrm{H}] \sim-0.4 \mathrm{dex})$ than the UCD. As will be presented in an accompanying paper (Fahrion et al., in prep.), FCC 47 has a bi-modal GC population that contains both GCs with metallicities similar to the UCD and more metal-rich GCs. Together with the short dynamical friction timescale, the low metallicity and blue colour are indications of an ex-situ origin of the UCD, irrespective of its GC or NSC nature.

Under the assumption that FCC 47-UCD1 was accreted during the assembly of the host galaxy, it is likely that it was the most massive star cluster of its original host galaxy. As Hilker (2009) showed, the absolute magnitude of the brightest GC or UCD in a galaxy scales with the host luminosity and total number of GCs. FCC 47-UCD1 falls among this relation as the brightest GC of FCC 47's rich GC system, but if it came from an accreted satellite, this dwarf galaxy must have had a smaller number of GCs than FCC 47 has today and thus it is difficult to reconcile the brightness of the UCD with a much smaller GC system of such a hypothetical progenitor following the relation described in Hilker (2009). However, this discrepancy would be naturally solved if FCC 47-UCD1 were not a nominal GC of its progenitor host, but instead the NSC; similar for example to M 54, which is considered to be the metalpoor NSC of the Sagittarius dwarf galaxy (Ibata et al. 1997; Bellazzini et al. 2008). At these low galaxy masses, colours and metallicities of dwarf nuclei are no longer distinguishable from blue GCs or UCDs (Norris \& Kannappan 2011; Sandoval et al. 2015; Spengler et al. 2017). Using HST/ACS, Georgiev et al. (2009b) studied the GC populations of late-type dwarf galaxies and found many massive star clusters with magnitudes $M_{V}<$ -10 mag. These massive GCs are found at small projected distances in the nuclear regions of these dwarf galaxies and show similar properties concerning the mass, size, and metallicity as M 54 or $\omega$ Cen. In the case that FCC 47-UCD1 was accreted, it is possible that it was the nuclear cluster of such a metal-poor dwarf satellite galaxy.

\subsection{Ultra compact dwarf projected distance}

The UCD in FCC 47 is special in two ways: it is found with a very close projected distance to the centre of FCC 47 and it is bound to a rather low-mass host. Nowadays, $>300$ UCDs are known. Most of them were found using large-scale imaging surveys combined with spectroscopic follow-up observations of the centres of galaxy clusters such as Virgo (Brodie et al. 2011; Liu et al. 2015), Fornax (Haşegan et al. 2005; Mieske et al. 2004), or even the Coma cluster (Price et al. 2009). Only a few were found around group or isolated galaxies, such as Centaurus A (Rejkuba et al. 2007) or the Sombrero galaxy (Hau et al. 2009; Norris \& Kannappan 2011). Figure 10 shows a comprehensive, but probably not complete collection of the currently known UCDs with their absolute $V$-band magnitudes versus projected distances to the assumed hosts. We describe in Appendix B how this sample was compiled. This figure reveals that there are only a few UCDs with distances $<5 \mathrm{kpc}$ from their host. This is most likely dominated by a selection effect because searches for UCDs are easier in the outskirts of galaxies where the local surface brightness is low.

Norris \& Kannappan (2011) reported on the discovery of a massive UCD close to the centre of the early-type S0 galaxy NGC 4546 using archival HST data in addition to Spectrographic Areal Unit for Research on Optical Nebulae (SAURON) IFU data. Although NGC 4546 is an isolated galaxy, the two systems are quite similar. Both FCC 47 and NGC 4546 are earlytype galaxies with masses of $\sim 2 \times 10^{10} M_{\odot}$ (Norris \& Kannappan 2011) and their UCDs were found at close projected distances 


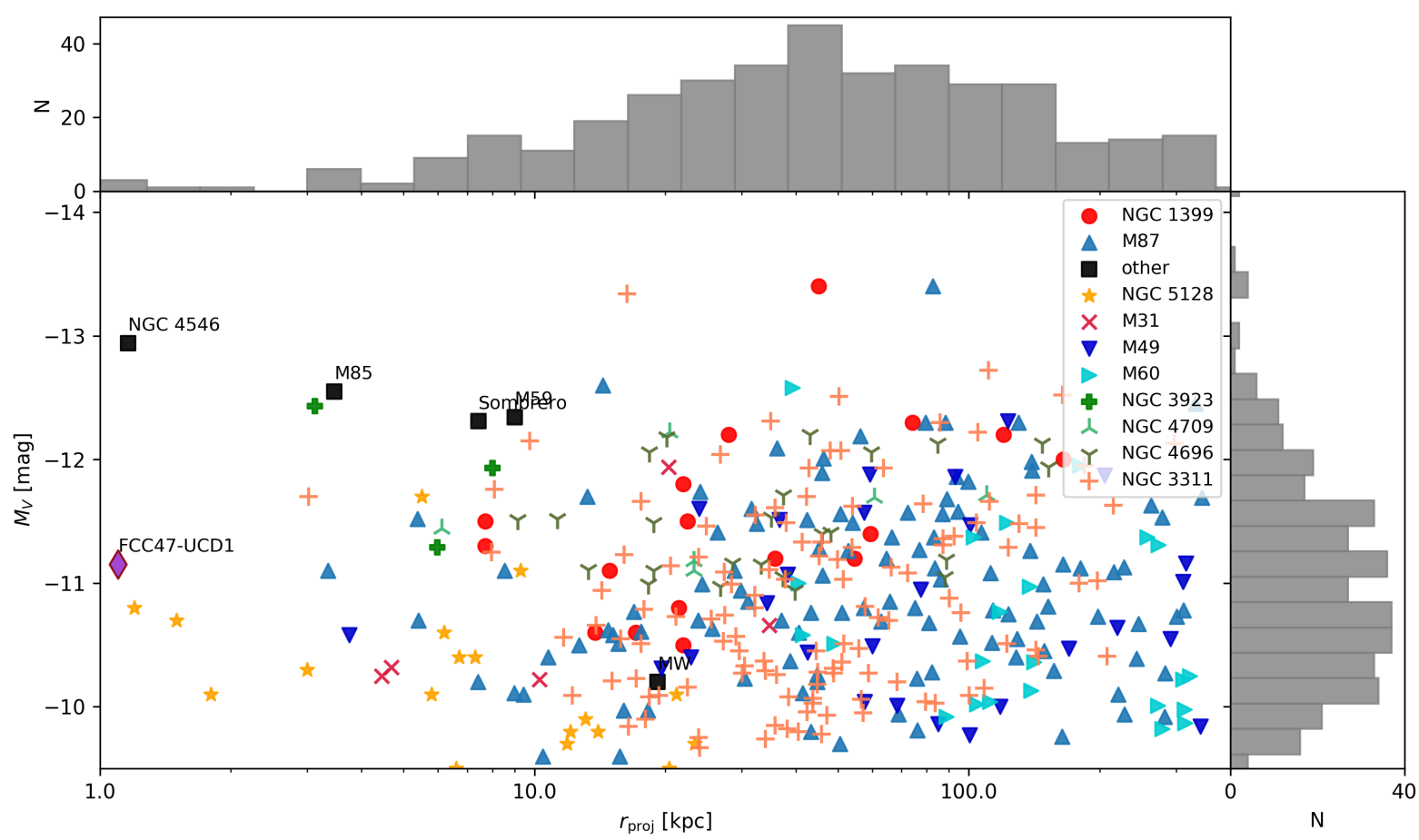

Fig. 10. Projected distances of UCDs to their assumed host galaxies versus their magnitudes. We show the UCDs from several sources including Rejkuba et al. (2007), Mieske et al. (2008), Brodie et al. (2011), Norris \& Kannappan (2011), Liu et al. (2015), Misgeld et al. (2011) and (Galleti et al. 2004). We show the sources with absolute magnitudes between $-9.5>M_{V}>-14.0$ mag, the magnitude range for typical UCDs. Details on how the data were compiled can be found in Appendix B.

of $\sim 1.1 \mathrm{kpc}$. However, with a total stellar mass of $\sim 3 \times 10^{7} M_{\odot}$ NGC 4546-UCD1 is much more massive than FCC 47-UCD1, but has a similar size $\left(R_{\mathrm{eff}} \sim 25 \mathrm{pc}\right)$. This is also reflected in its magnitude $\left(M_{V} \sim-13 \mathrm{mag}\right)$ and super-solar metallicity ([M/H] $\sim 0.2$ dex $)$. At a distance of $\sim 13 \mathrm{Mpc}$, deep spectroscopic observations with the Gemini Multi-Object Spectrographs (GMOS) were possible that enabled the discovery of an extended SFH Norris et al. (2015), although no central SMBH could be detected. The large magnitude gap to the GC system in addition to the extended SFH confirmed this object to be the remnant nucleus of a stripped dwarf galaxy.

The location of FCC 47-UCD1 in close projected distance to FCC 47's NSC is also reminiscent of the two-nucleus set-up found in the massive merger galaxy NGC 7727 (Schweizer et al. 2018). While the primary nucleus seems to fit well into the central luminosity and colour profile, the authors argue that the second nucleus is the stripped remnant of a former companion galaxy and therefore gives direct evidence for the formation of a UCD through stripping. The projected separation between the two nuclei $\left(r_{\text {proj }}=0.48 \mathrm{pc}\right)$ is even smaller than in FCC 47; however, there are obvious differences between the two systems: NGC 7727 is much more massive $\left(M_{*} \sim 1.4 \times 10^{11} M_{\odot}\right)$ than FCC 47 and has a complex structure due to mergers. With a $V$-band magnitude of $M_{V}=-15.5 \mathrm{mag}$, the second nucleus of NGC 7727 is much brighter than typical massive UCDs and might therefore reflect a young UCD in the formation process. Although the second nucleus in NGC 7727 is much more massive than FCC 47-UCD1, it is possible that the NGC 7727 system resembles what happened to FCC 47 and its UCD.

\subsection{Implications for other galaxies}

By construction, the searches for UCDs are biased towards large distances from the host galaxies, where the galaxy background is faint. FCC 47-UCD1 is the exception that confirms the rule as it is visible in both the HST data from the ACSFCS and the VST data used in the FDS, but was so far overlooked because of its small separation to FCC 47. One might ask whether the typically large UCD-host-separations are purely due to selection effects in the search, or whether this is rooted in their formation and evolution.

Studying the nature of the, on average, large separation of UCDs from their host galaxies, as compared to GCs, could also help to further constrain their nature as pure star cluster objects or remnant nuclei of dwarf galaxies. As more massive galaxies can form more massive star clusters (Schulz et al. 2016), starcluster UCDs should be found in these high mass galaxies, but at large separations because at smaller separations they would quickly merge into the centre because their native orbits would lead to small dynamical friction timescales. On the other hand, the remnant nuclei of stripped dwarf galaxies could also be found at smaller separations if the dwarf galaxy had a radial (or polar) orbit with respect to the host galaxy and is now near its pericentre distance or on a circularized orbit. Studying the presence of UCDs (and star clusters) with close projected distance to their host's centre can also help to constrain the underlying density profile of the host because cored profiles can stall dynamical friction significantly (Goerdt et al. 2006; Read et al. 2006; Cole et al. 2012).

The fact that the UCD in FCC 47 is bound to a rather lowmass host is also of interest. Typically, UCDs have been found in the centres of galaxy clusters and are by default associated to the most massive galaxy in the cluster (e.g. Hilker et al. 2007; Brodie et al. 2011; Liu et al. 2015). Only a few UCDs have been found that are associated with a more isolated galaxy such as NGC 4546 or the Sombrero galaxy (Norris \& Kannappan 2011).

While it was time consuming in the past to first find a UCD candidate in large-scale photometric data and then to confirm its 
nature with a spectrum, integral field spectroscopy with instruments like MUSE could potentially reveal a large number of UCDs even in the high surface brightness regions of galaxies, as was the case for FCC 47-UCD1. This could give insight into the radial distribution of UCDs and into possible correlations with host properties such as mass and morphology.

\section{Conclusions}

In this paper we report on the discovery of a UCD near the centre of the early-type galaxy FCC 47 using AO-supported MUSE WFM SV and archival HST data. We present a photometric analysis of the structure of this UCD and an analysis of its integrated MUSE spectrum. From combining those data and results we estimate the dynamical and stellar population mass of the UCD. We summarize our findings and their implications as follows:

- The UCD lies at a very close projected distance $(1.1 \mathrm{kpc})$ from the centre of FCC 47. It is the second known UCD at such close projected distance to its host centre. This might be partly due to observational biases in searches of UCDs around high surface brightness galaxies or due to very short dynamical friction timescales. Assuming a circular orbit in an isothermal sphere, FCC 47-UCD1 should fall into the NSC within the next 0.5 Gyr. This might imply that similarly massive and close UCDs might have already merged with their host galaxy nucleus, although a radial orbit significantly decreases the timescale and FCC 47 seems to be a triaxial galaxy.

- The UCD structure is fitted in 2D with IMFIT, finding that the best representation of the data is achieved with a single generalized King profile with an effective radius of $\sim 24 \mathrm{pc}$ (see Table 2). With this size, the UCD is more than twice as large as any $\mathrm{GC}$ in FCC 47.

- The UCD has a colour of $(g-z)=1.46$ and is not distinct from FCC 47's blue GC population. Its absolute magnitude of $M_{g}=-10.55 \mathrm{mag}$ is consistent with the high-luminosity end of FCC 47's GC population, but is also comparable to magnitudes of known NSCs of dwarf elliptical galaxies in the Fornax cluster.

- From the MUSE spectrum, we find a relative velocity of $\sim 65 \mathrm{~km} \mathrm{~s}^{-1}$ confirming membership of the FCC 47 system and an upper limit of the velocity dispersion of $\sigma<$ $17 \mathrm{~km} \mathrm{~s}^{-1}$. The inferred limit on the dynamical mass is $M_{\text {dyn }}<1.3^{+1.6} \times 10^{7} M_{\odot}$ and we find a upper limit on the dynamical mass-to-light ratio of $M / L_{\mathrm{dyn}}<6.47$.

- We find a spectroscopic metallicity of $[\mathrm{M} / \mathrm{H}]=$ $-1.12 \pm 0.10$ dex and a generally old age ( $>8$ Gyr). Our data do not give any indication of multiple or young populations. We can exclude a significant contribution to the spectrum from a young stellar population.

- From the spectroscopic metallicity, we determine the stellar population mass of $M_{\mathrm{SSP}}=4.87 \times 10^{6} M_{\odot}$. This mass can be considered the lower limit of the total mass.

- The low metallicity, old age, and magnitude make this UCD consistent with being the most massive, blue GC in FCC 47's large GC system. The blue colour, low metallicity compared to FCC 47 stellar body, and red GC population and dynamical arguments suggest an ex-situ origin. Although we cannot unambiguously confirm its nature, FCC 47-UCD1 could be a candidate for being the remnant NSC of a stripped metal-poor dwarf galaxy.

- In the case the UCD is the remnant NSC of a stripped dwarf galaxy, we use known scaling relations to estimate that its hypothetical progenitor could have been a dwarf galaxy with a mass of a few $10^{8} M_{\odot}$. This would imply that the progenitor has lost at least $95 \%$ of its initial mass in a minor merger event with a mass ratio of $\sim 1: 100$.

There are open questions that would need an answer to further characterize the UCD progenitor and the UCD itself. Additional data are needed to determine the true velocity dispersion of the UCD and in turn determine its true dynamical mass. For this, a spectrum with high $\mathrm{S} / \mathrm{N}$ and high spectral resolution is needed. In case the elevated dynamical mass-to-light ratio remains, its origin should be investigated. While variations in the IMF might be discovered with a high $\mathrm{S} / \mathrm{N}$ spectrum in the optical or infrared wavelength range, a detection of a central $\mathrm{SMBH}$ would need AO-supported IFU observations. Unfortunately, at the distance of the Fornax cluster, this UCD is probably too faint to be observed with current high angular resolution IFU instruments.

The serendipitous discovery of this UCD shows how powerful the MUSE instrument is with its wide field of view and high spatial resolution. Now, with the commissioning of the GALACSI AO system, this power to discover new objects has further increased because with $\mathrm{AO}$ one can not only achieve superb resolution and in turn detect fainter and more distant UCDs, but the system is also capable of acquiring good quality data under less than ideal conditions.

Acknowledgements. We want to thank the anonymous referee for comments that helped to improve this manuscript. This work is based on observations collected at the European Organization for Astronomical Research in the Southern Hemisphere under ESO programme 60.A-9192. KF is grateful to Dimitri Gadotti and Enrica Iodice for their contributions to the original MUSE SV proposal and especially to Lodovico Coccato who kindly did the data reduction of the MUSE + AO data. GvdV acknowledges funding from the European Research Council (ERC) under the European Union's Horizon 2020 research and innovation programme under grant agreement No 724857 (Consolidator Grant ArcheoDyn). E.M.C. is funded by Padua University through grants DOR1699945/16, DOR1715817/17, DOR1885254/18, and BIRD164402/16. RMcD is the recipient of an Australian Research Council Future Fellowship (project number FT150100333).

\section{References}

Afanasiev, A. V., Chilingarian, I. V., Mieske, S., et al. 2018, MNRAS, 477, 4856 Ahn, C. P., Seth, A. C., den Brok, M., et al. 2017, ApJ, 839, 72

Ahn, C. P., Seth, A. C., Cappellari, M., et al. 2018, ApJ, 858, 102

Bacon, R., Accardo, M., Adjali, L., et al. 2010, in Ground-based and Airborne Instrumentation for Astronomy III, Proc. SPIE, 7735, 773508

Barmby, P., McLaughlin, D. E., Harris, W. E., Harris, G. L. H., \& Forbes, D. A. 2007, AJ, 133, 2764

Baumgardt, H. 2017, MNRAS, 464, 2174

Baumgardt, H., \& Hilker, M. 2018, MNRAS, 478, 1520

Bekki, K., Couch, W. J., Drinkwater, M. J., \& Shioya, Y. 2003, MNRAS, 344, 399

Bell, E. F., \& de Jong, R. S. 2001, ApJ, 550, 212

Bellazzini, M., Ibata, R. A., Chapman, S. C., et al. 2008, AJ, 136, 1147

Binney, J., \& Tremaine, S. 1987, Galactic Dynamics (Princeton: Princeton University)

Blakeslee, J. P., Jordán, A., Mei, S., et al. 2009, ApJ, 694, 556

Brodie, J. P., Romanowsky, A. J., Strader, J., \& Forbes, D. A. 2011, AJ, 142, 199 Cappellari, M. 2017, MNRAS, 466, 798

Cappellari, M., \& Emsellem, E. 2004, PASP, 116, 138

Cenarro, A. J., Cardiel, N., Gorgas, J., et al. 2001, MNRAS, 326, 959

Chilingarian, I. V., Mieske, S., Hilker, M., \& Infante, L. 2011, MNRAS, 412, 1627

Cole, D. R., Dehnen, W., Read, J. I., \& Wilkinson, M. I. 2012, MNRAS, 426, 601

Côté, P., Marzke, R. O., \& West, M. J. 1998, ApJ, 501, 554

Côté, P., Piatek, S., Ferrarese, L., et al. 2006, ApJS, 165, 57

Couture, J., \& Hardy, E. 1988, JRASC, 82, 277

Dabringhausen, J., Kroupa, P., \& Baumgardt, H. 2009, MNRAS, 394, 1529

Da Rocha, C., Mieske, S., Georgiev, I. Y., et al. 2011, A\&A, 525, A86

de Marchi, G. 1999, AJ, 117, 303

Dolphin, A. E. 2009, PASP, 121, 655 
Drinkwater, M. J., Jones, J. B., Gregg, M. D., \& Phillipps, S. 2000, PASA, 17, 227

Drinkwater, M. J., Gregg, M. D., Hilker, M., et al. 2003, Nature, 423, 519

Du, M., Debattista, V. P., Ho, L. C., et al. 2019, ApJ, 875, 58

Eigenthaler, P., Puzia, T. H., Taylor, M. A., et al. 2018, ApJ, 855, 142

Erwin, P. 2015, ApJ, 799, 226

Evstigneeva, E. A., Gregg, M. D., Drinkwater, M. J., \& Hilker, M. 2007a, AJ, 133,1722

Evstigneeva, E. A., Drinkwater, M. J., Jurek, R., et al. 2007b, MNRAS, 378, 1036

Evstigneeva, E. A., Drinkwater, M. J., Peng, C. Y., et al. 2008, AJ, 136, 461

Fellhauer, M., \& Kroupa, P. 2002, MNRAS, 330, 642

Fellhauer, M., \& Kroupa, P. 2005, MNRAS, 359, 223

Ferguson, H. C. 1989, AJ, 98, 367

Ferrarese, L., Côté, P., Dalla Bontà, E., et al. 2006, ApJ, 644, L21

Forbes, D. A., Norris, M. A., Strader, J., et al. 2014, MNRAS, 444, 2993

Francis, K. J., Drinkwater, M. J., Chilingarian, I. V., Bolt, A. M., \& Firth, P. 2012, MNRAS, 425, 325

Freudling, W., Romaniello, M., Bramich, D. M., et al. 2013, A\&A, 559, A96

Gallazzi, A., Charlot, S., Brinchmann, J., White, S. D. M., \& Tremonti, C. A 2005, MNRAS, 362, 41

Galleti, S., Federici, L., Bellazzini, M., Fusi Pecci, F., \& Macrina, S. 2004, A\&A, 416, 917

Georgiev, I. Y., Goudfrooij, P., Puzia, T. H., \& Hilker, M. 2008, AJ, 135, 1858

Georgiev, I. Y., Hilker, M., Puzia, T. H., Goudfrooij, P., \& Baumgardt, H. 2009a, MNRAS, 396, 1075

Georgiev, I. Y., Puzia, T. H., Hilker, M., \& Goudfrooij, P. 2009b, MNRAS, 392, 879

Georgiev, I. Y., Böker, T., Leigh, N., Lützgendorf, N., \& Neumayer, N. 2016, MNRAS, 457, 2122

Girardi, L., Bressan, A., Bertelli, G., \& Chiosi, C. 2000, A\&AS, 141, 371

Glass, L., Ferrarese, L., Côté, P., et al. 2011, ApJ, 726, 31

Goerdt, T., Moore, B., Read, J. I., Stadel, J., \& Zemp, M. 2006, MNRAS, 368 1073

Graham, A. W., \& Driver, S. P. 2007, ApJ, 655, 77

Graham, A. W., \& Spitler, L. R. 2009, MNRAS, 397, 2148

Guérou, A., Krajnović, D., Epinat, B., et al. 2017, A\&A, 608, A5

Harris, W. E. 1996, AJ, 112, 1487

Harris, W. E., Harris, G. L. H., \& Alessi, M. 2013, ApJ, 772, 82

Haşegan, M., Jordán, A., Côté, P., et al. 2005, ApJ, 627, 203

Hau, G. K. T., Spitler, L. R., Forbes, D. A., et al. 2009, MNRAS, 394, L97

Hilker, M. 2009, Rev. Mod. Astron., 21, 199

Hilker, M., \& Richtler, T. 2000, A\&A, 362, 895

Hilker, M., Infante, L., Vieira, G., Kissler-Patig, M., \& Richtler, T. 1999a, A\&AS, 134,75

Hilker, M., Infante, L., \& Richtler, T. 1999b, A\&AS, 138, 55

Hilker, M., Baumgardt, H., Infante, L., et al. 2007, A\&A, 463, 119

Huxor, A. P., Ferguson, A. M. N., Tanvir, N. R., et al. 2011, MNRAS, 414, 770

Ibata, R. A., Wyse, R. F. G., Gilmore, G., Irwin, M. J., \& Suntzeff, N. B. 1997, AJ, 113, 634

Iodice, E., Capaccioli, M., Grado, A., et al. 2016, ApJ, 820, 42

Janz, J., Norris, M. A., Forbes, D. A., et al. 2016, MNRAS, 456, 617

Jordán, A., Blakeslee, J. P., Peng, E. W., et al. 2004, ApJS, 154, 509

Jordán, A., Blakeslee, J. P., Côté, P., et al. 2007, ApJS, 169, 213

Jordán, A., Peng, E. W., Blakeslee, J. P., et al. 2009, ApJS, 180, 54

Jordán, A., Peng, E. W., Blakeslee, J. P., et al. 2015, ApJS, 221, 13

King, I. R., Bedin, L. R., Cassisi, S., et al. 2012, AJ, 144, 5

Kirby, E. N., Cohen, J. G., Guhathakurta, P., et al. 2013, ApJ, 779, 102

Kissler-Patig, M., Jordán, A., \& Bastian, N. 2006, A\&A, 448, 1031

Koleva, M., Prugniel, P., De Rijcke, S., \& Zeilinger, W. W. 2011, MNRAS, 417, 1643

Kormendy, J., \& Ho, L. C. 2013, ARA\&A, 51, 511

Lequeux, J., Peimbert, M., Rayo, J. F., Serrano, A., \& Torres-Peimbert, S. 1979, A\&A, 500, 145

Liu, C., Peng, E. W., Côté, P., et al. 2015, ApJ, 812, 34

Liu, Y., Peng, E. W., Lim, S., et al. 2016, ApJ, 830, 99

Lotz, J. M., Miller, B. W., \& Ferguson, H. C. 2004, ApJ, 613, 262

Ma, J., Wang, S., Wu, Z., et al. 2017, MNRAS, 468, 4513

Magorrian, J., Tremaine, S., Richstone, D., et al. 1998, AJ, 115, 2285

Majewski, S. R., Patterson, R. J., Dinescu, D. I., et al. 2000, in Liege Internationa Astrophysical Colloquia, eds. A. Noels, P. Magain, D. Caro, et al., 35, 619

Maraston, C., Bastian, N., Saglia, R. P., et al. 2003, IAU Jt. Discuss., 25, E18

Maraston, C., Bastian, N., Saglia, R. P., et al. 2004, A\&A, 416, 467

McLaughlin, D. E., \& van der Marel, R. P. 2005, A\&AS, 161, 304

Mendel, J. T., Proctor, R. N., Rasmussen, J., Brough, S., \& Forbes, D. A. 2009 MNRAS, 396, 2103
Meylan, G., Sarajedini, A., Jablonka, P., et al. 2001, AJ, 122, 830

Mieske, S., \& Kroupa, P. 2008, ApJ, 677, 276

Mieske, S., Hilker, M., \& Infante, L. 2002, A\&A, 383, 823

Mieske, S., Hilker, M., \& Infante, L. 2004, A\&A, 418, 445

Mieske, S., Hilker, M., Infante, L., \& Jordán, A. 2006, AJ, 131, 2442

Mieske, S., Hilker, M., Jordán, A., Infante, L., \& Kissler-Patig, M. 2007, A\&A, 472,111

Mieske, S., Hilker, M., Jordán, A., et al. 2008, A\&A, 487, 921

Mieske, S., Hilker, M., Misgeld, I., et al. 2009, A\&A, 498, 705

Mieske, S., Frank, M. J., Baumgardt, H., et al. 2013, A\&A, 558, A14

Minniti, D., Kissler-Patig, M., Goudfrooij, P., \& Meylan, G. 1998, AJ, 115, 121

Misgeld, I., \& Hilker, M. 2011, MNRAS, 414, 3699

Misgeld, I., Mieske, S., Hilker, M., et al. 2011, A\&A, 531, A4

Murray, N. 2009, ApJ, 691, 946

Neumayer, N., \& Walcher, C. J. 2012, Adv. Astron., 2012, 709038

Norris, M. A., \& Kannappan, S. J. 2011, MNRAS, 414, 739

Norris, M. A., Kannappan, S. J., Forbes, D. A., et al. 2014, MNRAS, 443, 1151 Norris, M. A., Escudero, C. G., Faifer, F. R., et al. 2015, MNRAS, 451, 3615

Ordenes-Briceño, Y., Puzia, T. H., Eigenthaler, P., et al. 2018, ApJ, 860, 4

Paudel, S., Lisker, T., \& Kuntschner, H. 2011, MNRAS, 413, 1764

Peng, E. W., Jordán, A., Côté, P., et al. 2006, ApJ, 639, 95

Pfeffer, J., \& Baumgardt, H. 2013, MNRAS, 433, 1997

Pfeffer, J., Griffen, B. F., Baumgardt, H., \& Hilker, M. 2014, MNRAS, 444, 3670

Pfeffer, J., Hilker, M., Baumgardt, H., \& Griffen, B. F. 2016, MNRAS, 458, 2492

Pfeffer, J., Kruijssen, J. M. D., Crain, R. A., \& Bastian, N. 2018, MNRAS, 475, 4309

Phillipps, S., Drinkwater, M. J., Gregg, M. D., \& Jones, J. B. 2001, ApJ, 560, 201

Pietrinferni, A., Cassisi, S., Salaris, M., \& Castelli, F. 2004, ApJ, 612, 168

Pietrinferni, A., Cassisi, S., Salaris, M., \& Castelli, F. 2006, ApJ, 642, 797

Price, J., Phillipps, S., Huxor, A., et al. 2009, MNRAS, 397, 1816

Read, J. I., Goerdt, T., Moore, B., et al. 2006, MNRAS, 373, 145

Rejkuba, M., Dubath, P., Minniti, D., \& Meylan, G. 2007, A\&A, 469, 147

Ricciardelli, E., Vazdekis, A., Cenarro, A. J., \& Falcón-Barroso, J. 2012, MNRAS, 424, 172

Sandoval, M. A., Vo, R. P., Romanowsky, A. J., et al. 2015, ApJ, 808, L32

Sarzi, M., Iodice, E., Coccato, L., et al. 2018, A\&A, 616, A121

Saulder, C., van Kampen, E., Chilingarian, I. V., Mieske, S., \& Zeilinger, W. W. 2016, A\&A, 596, A14

Schulz, C., Hilker, M., Kroupa, P., \& Pflamm-Altenburg, J. 2016, A\&A, 594, A119

Schweizer, F., Seitzer, P., Whitmore, B. C., Kelson, D. D., \& Villanueva, E. V. 2018, ApJ, 853, 54

Seth, A., Agüeros, M., Lee, D., \& Basu-Zych, A. 2008, ApJ, 678, 116

Seth, A. C., van den Bosch, R., Mieske, S., et al. 2014, Nature, 513, 398

Sirianni, M., Jee, M. J., Benítez, N., et al. 2005, PASP, 117, 1049

Soto, K. T., Lilly, S. J., Bacon, R., Richard, J., \& Conseil, S. 2016, MNRAS, 458, 3210

Spengler, C., Côté, P., Roediger, J., et al. 2017, ApJ, 849, 55

Strader, J., Romanowsky, A. J., Brodie, J. P., et al. 2011, ApJS, 197, 33

Strader, J., Seth, A. C., Forbes, D. A., et al. 2013, ApJ, 775, L6

Taylor, M. A., Puzia, T. H., Harris, G. L., et al. 2010, ApJ, 712, 1191

Tremonti, C. A., Heckman, T. M., Kauffmann, G., et al. 2004, ApJ, 613, 898

Turner, M. L., Côté, P., Ferrarese, L., et al. 2012, ApJS, 203, 5

Vazdekis, A., Sánchez-Blázquez, P., Falcón-Barroso, J., et al. 2010, MNRAS, 404, 1639

Vazdekis, A., Ricciardelli, E., Cenarro, A. J., et al. 2012, MNRAS, 424, 157

Vazdekis, A., Koleva, M., Ricciardelli, E., Röck, B., \& Falcón-Barroso, J. 2016, MNRAS, 463, 3409

Villegas, D., Jordán, A., Peng, E. W., et al. 2010, ApJ, 717, 603

Voggel, K., Hilker, M., \& Richtler, T. 2016, in The General Assembly of Galaxy Halos: Structure, Origin and Evolution, eds. A. Bragaglia, M. Arnaboldi, M. Rejkuba, \& D. Romano, IAU Symp., 317, 365

Voggel, K. T., Seth, A. C., Neumayer, N., et al. 2018, ApJ, 858, 20

Walcher, C. J., van der Marel, R. P., McLaughlin, D., et al. 2005, ApJ, 618, 237

Weilbacher, P. M., Streicher, O., \& Palsa, R. 2016, Astrophysics Source Code Library [record ascl:1610.004]

Weilbacher, P. M., Streicher, O., Urrutia, T., et al. 2014, in Astronomical Data Analysis Software and Systems XXIII, eds. N. Manset, \& P. Forshay, ASP Conf. Ser., 485, 451

Wittmann, C., Lisker, T., Pasquali, A., Hilker, M., \& Grebel, E. K. 2016, MNRAS, 459, 4450

Yoon, S.-J., Lee, S.-Y., Blakeslee, J. P., et al. 2011, ApJ, 743, 150

Zinnecker, H., Keable, C. J., Dunlop, J. S., Cannon, R. D., \& Griffiths, W. K. 1988, in The Harlow-Shapley Symposium on Globular Cluster Systems in Galaxies, eds. J. E. Grindlay, \& A. G. D. Philip, IAU Symp., 126, 603 


\section{Appendix A: imfit and PPXF fit distributions}

We used IMFIT's capability to perform a MCMC analysis to determine the best-fitting model parameters. Figure A.1 shows a corner plot displaying the distribution of parameters from the MCMC IMFIT run on the F475W data with a single generalized King profile. Overall, the distributions are well defined and Gaussian, although there are small degeneracies between some parameters. However, the uncertainties on the best-fitting values are very small $(<1 \%)$, possibly because the UCD is very bright compared to the galaxy-subtracted background and the HST data is of high quality. To get more realistic errors, we performed IMFIT's bootstrapping analysis on the best-fit MCMC parameters with 1000 iterations. The resulting histograms are shown in Fig. A.2.

As described in Sect. 3, we created 600 realizations of the UCD MUSE spectrum and fitted it with PPXF using the EMILES SSP library to create well-sampled distributions for the radial velocity and mean metallicity. The distributions are shown in Fig. A.3.

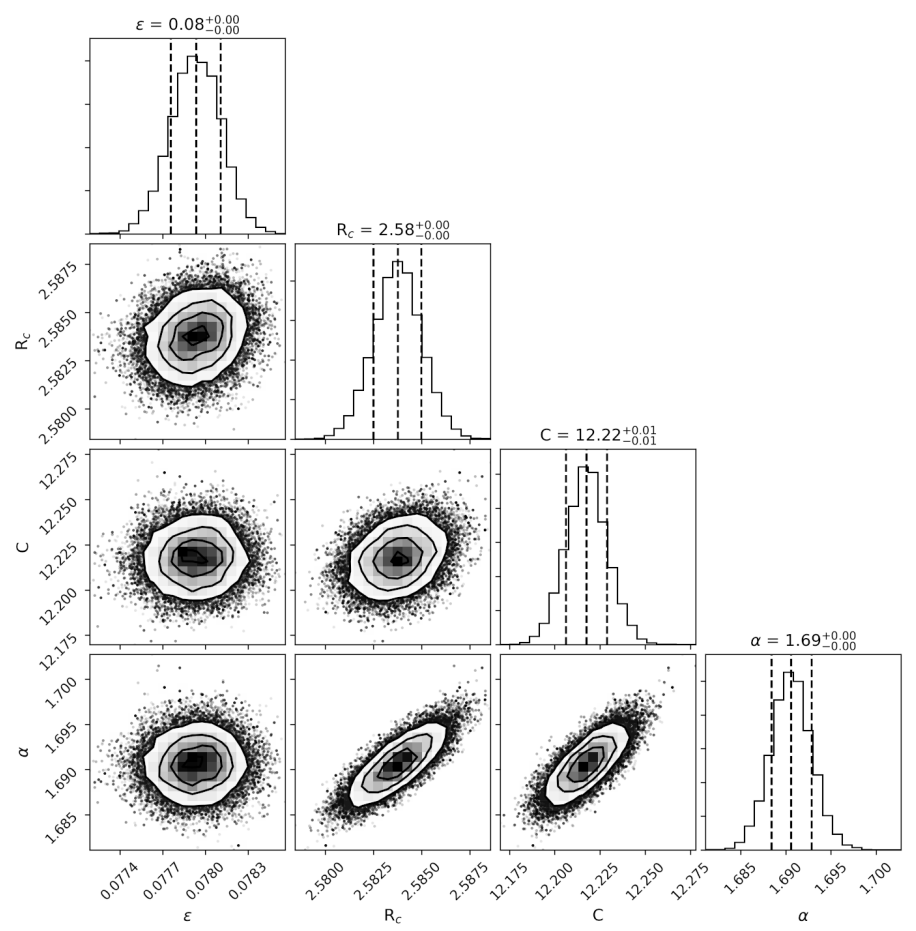

Fig. A.1. Corner plot showing the distribution of parameters for the MCMC IMFIT fit of the single generalized King profile to the F475W data.
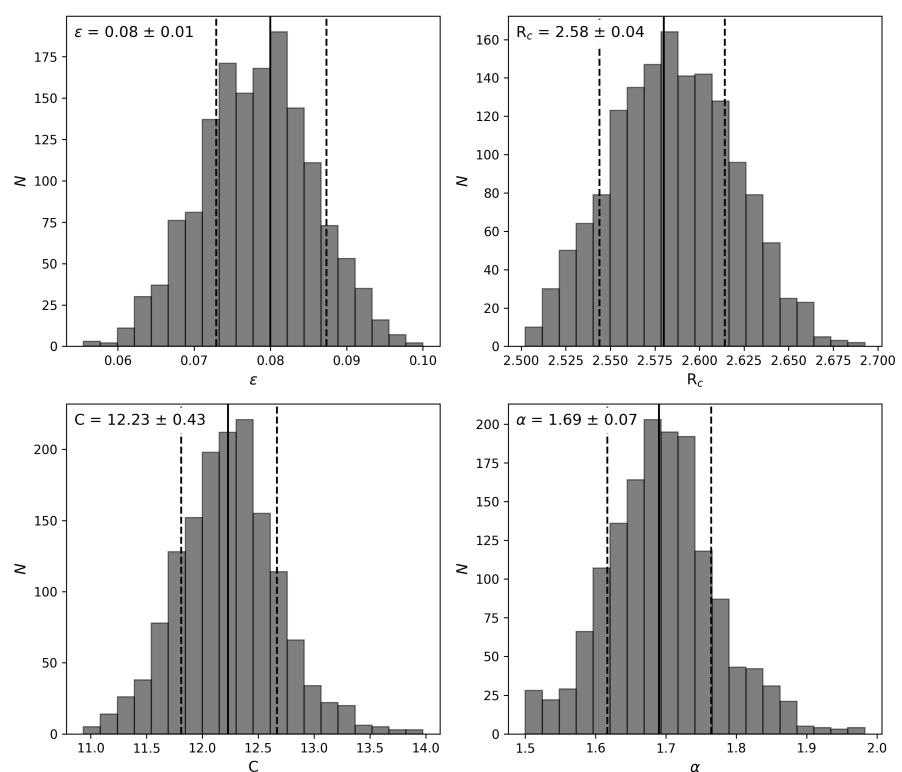

Fig. A.2. Histograms showing the results from bootstrapping around the MCMC best-fit parameters of the single generalized King profile fit to the $\mathrm{F} 475 \mathrm{~W}$ data. We use 1000 iterations.
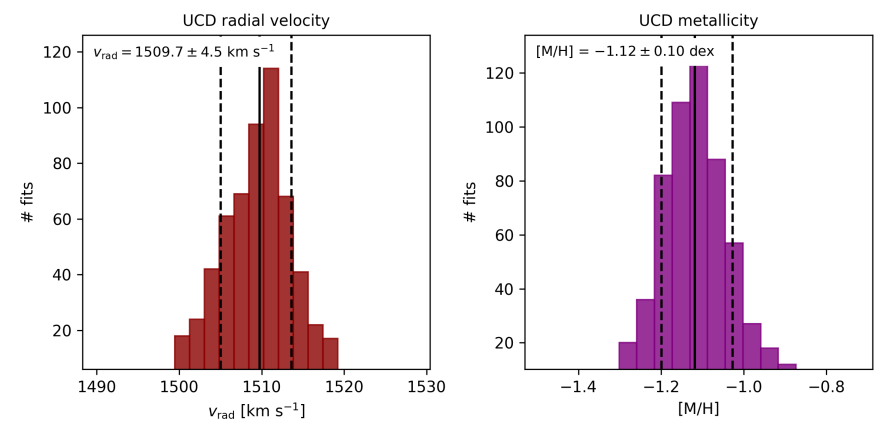

Fig. A.3. Radial velocity and mean metallicity distributions. These distributions were obtained by fitting 600 realizations of the UCD spectrum with PPXF in the full MUSE wavelength range. 


\section{Appendix B: Catalogue of literature UCDs}

For the purpose of comparing FCC 47-UCD1 with others, we have collected a sample of about 350 UCDs and other dense stellar systems from the literature. Our collection is taken from:

- Mieske et al. (2008). The authors have published a catalogue of 55 dense stellar systems around NGC 1399, M 87, CenA, and others. The references of these sources are Haşegan et al. (2005), Hilker et al. (2007), Rejkuba et al. (2007), Evstigneeva et al. (2007a), McLaughlin \& van der Marel (2005), Meylan et al. (2001), Barmby et al. (2007), de Marchi (1999). Most of these sources probably are UCDs, but they also include GCs around CenA.

- Brodie et al. (2011). Their sample includes 25 UCDs around M 87.

- Liu et al. (2015). Their sample includes 142 UCDs around M 87, M 49, and M 60. Some of the sources associated with M 60 might truly be hosted by M 59. The authors report $g$ band magnitudes and $(u-g)$ colours that we convert to $V$ band using the relations from Lupton ${ }^{2}$.

- Norris \& Kannappan (2011). The authors have discovered three UCDs around NGC 3923, one around NGC 4546, and study one around the Sombrero galaxy (M 104), first discovered by Hau et al. (2009).

- Price et al. (2009). The sample contains six massive compact objects in the Coma galaxy cluster. Three of them are clearly identified as cE galaxies.

- Evstigneeva et al. (2007b). This includes three UCDs around Dorado and two around NGC 1400.
- Misgeld et al. (2011). Their catalogue includes 118 members of the Hydra I galaxy cluster, 52 of which are bright enough to be considered to be UCDs.

- Mieske et al. (2007, 2009). The sample includes 30 compact objects found in the Centaurus galaxy cluster. Six of the objects are associated with the Cen 45 sub-cluster dominated by NGC 4709 while the rest are associated to Cen30 (NGC 4696).

- M31: We select the brightest $\left(M_{V}<-10.0\right)$ GCs from the revised Bologna Catalogue (Galleti et al. 2004) as well as the extended clusters found around M31 from Huxor et al. (2011).

We further add GC-2 as a UCD around M 81 (Ma et al. 2017), W3 in NGC 7252 (Maraston et al. 2003). We remove double entries from the sample and calculate the projected distances to their assumed host galaxy if coordinates are given. For this and for the calculation of absolute magnitudes, we use distances either from the quoted works directly or from the NED database $^{3}$. We have half-light radii and absolute $V$-band magnitudes (only $B$-band magnitudes for the sources from Price et al. 2009) for almost all sources and have included the radial velocities for 300 UCDs. Almost all UCDs have an assigned host from the literature. Mostly, it is the central galaxy of the field that was observed and sometimes it is the closest in projection. We do not test how likely the association is, but give for each host galaxy basic properties such as sky coordinates, magnitudes, and heliocentric radial velocities. We show the catalogue in Table B.1 in a shortened form. The full catalogue is available at the CDS.

Table B.1. Catalogue of literature ultra compact dwarf galaxies in shortened form.

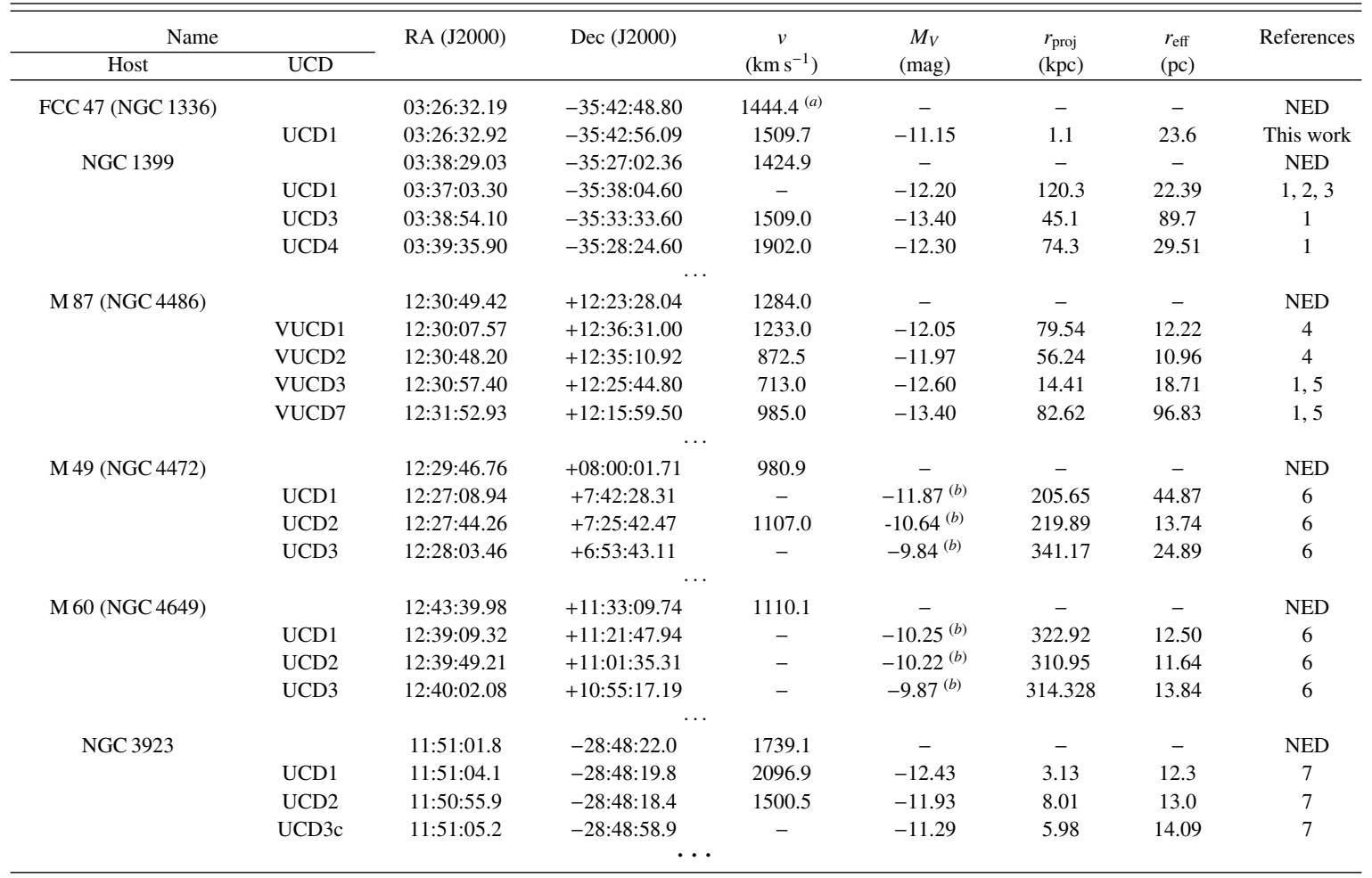

Notes. ${ }^{(a)}$ From Fahrion et al., in prep. ${ }^{(b)}$ Converted from $g$-band magnitudes and $(u-g)$ colours. The full catalogue is available at the CDS.

References. (1) Mieske et al. (2008), (2) Hilker et al. (2007), (3) Drinkwater et al. (2003), (4) Brodie et al. (2011), (5) Evstigneeva et al. (2007a), (6) Liu et al. (2015), (7) Norris \& Kannappan (2011).

\footnotetext{
wWW.sdss.org/dr7/algorithms/sdssUBVRITransform.html
}

3 https://ned.ipac.caltech.edu/ 